\title{
Inhibition of Methamphetamine Self-Administration and Reinstatement by Central Blockade of Angiotensin II Receptor in Rats ${ }^{[\Phi]}$
}

\author{
Xing $\mathrm{Xu}$, Jian Pan, Xingxing Li, Yan Cui, Zijuan Mao, Boliang Wu, Huachong Xu, \\ Wenhua Zhou, and Yu Liu
}

Ningbo University School of Medicine, Ningbo, Zhejiang, People's Republic of China (X.X., J.P., Z.M., B.W., W.Z., Y.L.); Ningbo Kangning Hospital, Ningbo, Zhejiang, People's Republic of China (X.L.); Ningbo Public Security Bureau Ningbo Anti-drug Office, Zhejiang, People's Republic of China (Y.C., H.X.); and Ningbo Addiction Research and Treatment Center, Zhejiang, People's Republic of China (W.Z.)

Received December 13, 2018; accepted March 4, 2019

\section{ABSTRACT}

The molecular mechanism and treatment of methamphetamine (METH) use disorder remain unclear. The current study aimed to investigate the role of central angiotensin II receptor (ATR) in drug taking and seeking behavior associated with METH use disorder. The effect of an ATR type 1 (AT1R) antagonist, candesartan cilexetil, on the reinforcing and motivational effects of METH was first assessed using the animal model of METH self-administration (SA) and reinstatement. The levels of dopamine D2 receptor (D2R) and AT1R were subsequently examined. Furthermore, the present study determined the expression of microRNAs (miRNAs) by comparing METH SA, METH-yoked, and Saline-yoked groups. The target miRNAs were further overexpressed in the nucleus accumbens (NAc) via a lentivirus vector to investigate the effects of target miRNAs on METH SA maintained under a fixed ratio 1 , progressive ratio, and cue/drug reinstatement of METHSA. The potential role of the AT1R-PLC $\beta$ CREB signaling pathway was finally investigated. The results suggest that AT1R blockade effectively reduced METH SA and reinstatement, in conjunction with the counter-regulation of D2R and AT1R. A total of 17 miRNAs targeting Ang II in NAc were found to be associated with the voluntary intake of METH. Furthermore, overexpression of specific miR-219a-5p targeting AT1R-regulated METH SA and reinstatement. The AT1R-PLC $\beta$ CREB signaling pathway was found to be associated with the effect of AT1R on the drug-taking and drug-seeking behavior involving METH use disorder.

\section{Introduction}

Methamphetamine (METH) use disorder has resulted in major public health problems worldwide (Fulcher et al., 2018; Gao et al., 2018; Krizman-Matasic et al., 2019). In addition to compulsive drug-taking and drug-seeking behavior, individuals with METH use disorder commonly present with other comorbidities, including cognitive deficits, depression, psychosis, anxiety, and sleep disorders (Grant et al., 2012; McKetin et al., 2013; Mullen and Crawford, 2018). These comorbid disorders may further add to the complex consequences of METH use disorder, supporting the need for "transdiagnostic treatment approaches" (Bernheim et al., 2016;

This work was supported in part by the National Natural Science Foundation of China (Grant 81671323), the National Natural Science Foundation of Ningbo (Grant 2015A610188), the K. C. Wong Magna Fund in Ningbo University, Zhejiang Provincial Key Laboratory of Pathophysiology (Ningbo University School of Medicine), and the Program for Innovative Research Team in Ningbo City (Grant 2015C110026)

https://doi.org/10.1124/jpet.118.255729.

S This article has supplemental material available at jpet.aspetjournals.org.
Hartel-Petri et al., 2017). Given that no medications have demonstrated a convincing and consistent effect on the treatment of METH use disorder, an increasing number of studies have highlighted the potential use of medications targeting cognitive impairments associated with executive function for METH use disorder (Sofuoglu et al., 2013). Cholinesterase inhibitors (e.g., galantamine and rivastigmine) and monoamine transporter inhibitors (e.g., modafinil and methylphenidate) have shown promising effects on improvement of the treatment outcomes of METH use disorder (Sofuoglu et al., 2013; Franke et al., 2014).

Early studies demonstrated an independent renin-angiotensin system (RAS) in the central nervous system (Basmadjian et al., 2017). The central binding sites of Angiotensin II (Ang II) have been identified in the cortex, hippocampus (Hip), and midbrain (Wright and Harding, 1994; Holme et al., 2018). In the central nervous system, an abundant increase of Ang II is associated with neuroinflammation, neuronal damage, and neurodegeneration. Ang II has two different subtypes of cellsurface receptors, Ang II receptor type 1 (AT1R) and Ang II

ABBREVIATIONS: Ang II, angiotensin II; AT1R, angiotensin II receptor type 1; CAN, candesartan cilexetil; CPU, caudate putamen; CREB, cAMP responsive element-binding protein; DAG, diacylglycerol; D2R, dopamine D2 receptor; FR1, fixed ratio 1; Hip, hippocampus; IP3, inositol triphosphate; METH, methamphetamine; miRNA, microRNA; NAc, nucleus accumbens; PC12, pheochromocytoma 12; PFC, prefrontal cortex; PLC, phospholipase C; PR, progressive ratio; PVDF, polyvinylidene difluoride; RAS, renin-angiotensin system; RT-qPCR, quantitative real-time polymerase chain reaction; SA, self-administration. 
receptor type 2. Brain Ang II, mainly through AT1R, has also been demonstrated to interact with dopaminergic, glutamatergic, and GABAergic neurotransmission in the brain (Basmadjian et al., 2017). AT1R blockade has been suggested to be a therapeutic target for the cognitive deficits associated with a wide range of central nervous system disorders, including Alzheimer's disease, HIV infection, neuropathic pain, Parkinson's disease, schizophrenia, and depression (Muthuraman and Kaur, 2016; Saavedra, 2016; Zhuang et al., 2016; Erlandson et al., 2017; Fan et al., 2017; Perez-Lloret et al., 2017; Vian et al., 2017).

A limited number of studies have begun to explore the role of central Ang II and AT1R on the behavioral consequences of drugs of abuse. For example, AT1R in the central nucleus of the amygdala has been shown to involve the hemodynamic responses to cocaine in rats (Knuepfer et al., 2005; Watanabe et al., 2010). Blockade of AT1R by losartan enabled to elevate cocaine-induced hemodynamic responses in rats (Watanabe et al., 2010). Multiple doses of METH significantly unregulated the expression of AT1R in the striatum of mice, along with decreased dopamine D3 receptor expression (Jiang et al., 2018). The inhibition or genetic deletion of AT1R was sufficient to attenuate the hyperlocomotion induced by METH and amphetamine in mice (Jiang et al., 2018). An increased AT1R density induced by 21 days of amphetamine injections was evident in both caudate putamen (CPU) and NAc of mice. In contrast, the desensitized response of AT1R to Ang II has also been reported in rats with repeated injections of amphetamine (Casarsa et al., 2015). However, direct microinjection of Ang II in the rat ventral tegmental area (VTA) and NAc significantly lowered morphine self-administration (SA) (Hosseini et al., 2007, 2009). In addition, the injection of Ang II into the NAc failed to alter morphine-induced conditional place preference in rats (Hosseini et al., 2007).

The majority of the previously described studies have used passive administration of drugs of abuse and an animal model of behavioral sensitization. Drug use disorder is characterized by a compulsion to seek and use drugs, a loss of control over intake, and repeated episodes of relapse (Cami and Farre, 2003). Up to the present, the effects of central ATR1 and the Ang II system on the addictive properties of drugs have not been reported in the literature. The current study is designed to evaluate the role of Ang II and AT1R in the addictive aspects of METH, using the animal model of METH SA and cue-/drug-induced reinstatement. A specific microRNA (miRNA) that targeted AT1R was also identified in the current study. The potential role of the AT1R-phospholipase C (PLC) $\beta$-cAMP responsive element-binding protein (CREB) signaling pathway in the effect of AT1R blockade on METH was finally investigated.

\section{Materials and Methods}

\section{Animals}

Male Sprague-Dawley rats (Zhejiang Academy of Medical Sciences, Hangzhou, Zhejiang, People's Republic of China) weighing approximately $300-350 \mathrm{~g}$ at the beginning of the experiment were individually housed under a standard environment (temperature, $23 \pm 1{ }^{\circ} \mathrm{C}$; humidity, $40 \%-70 \%$ ) with a 12-hour light/dark cycle (lights off at 07:00 AM, on at 7:00 PM). Food and water were provided abundantly. The experimental protocol was approved by an Institutional Review Committee for the use of Animal Subjects. All the experiments were conducted in accordance with the guidelines of the Institutional Laboratory Animal Care and Use of Ningbo University.

\section{Drugs}

METH ( $>90 \%$ purity) was provided by the Ningbo Public Security Bureau Ningbo Anti-drug Office and dissolved in physiologic saline. The purity of METH was measured by high-performance liquid chromatography. The mobile phase contained $\mathrm{KH}_{2} \mathrm{PO}_{4}$ buffer ( $50 \mathrm{mmol} / \mathrm{l}, \mathrm{pH} 3.0$ ) and acetonitrile $(70: 30, \mathrm{v} / \mathrm{v} \%)$. The flow rate was $1 \mathrm{ml} / \mathrm{min}$. A Phenomenex (Torrance, CA) Luna $5 \mu \mathrm{m}$ SCX $100 \AA$ column was used $(250 \times 4.6 \mathrm{~mm}$, $5 \mu \mathrm{M})$. The measured wavelengths were $215 \mathrm{~nm}$. The wavelengths with a range of $190-360 \mathrm{~nm}$ were collected. The retention time was 11 minutes. The sample was dissolved in the mobile phase, and the concentration was $1.0 \mathrm{mg} / \mathrm{ml}$. The injection volume was $20 \mu \mathrm{l}$ for all measurements. The measurement of each sample was carried out six times. Candesartan cilexetil (CAN; TCV-116; Tokyo Chemical Industry, Tokyo, Japan) was dissolved in $100 \%$ DMSO and administered via an oral gavage route.

\section{Surgery}

The animals were anesthetized with $1.5 \%$ sodium pentobarbital $(30 \mathrm{mg} / \mathrm{kg})$ and $0.03 \mathrm{ml}$ xylazine hydrochloride. Each animal had a silastic catheter implanted in the jugular vein. The catheter was passed subcutaneously to a polyethylene assembly mounted on the back of the animal. The catheters were flushed daily with sterile saline containing heparin sodium $(0.4 \%)$ and penicillin sodium to maintain catheter patency and prevent infection.

\section{METH SA}

Rats were permitted at least 3 days of recovery from the surgery before behavioral training was conducted. Rats were trained to respond on an active hole to receive METH infusions. A nose-poke response in the left (active) hole was immediately reinforced with an injection of METH. A nose-poke response in the right (inactive) hole was considered as an inactive response, which had no programmed consequences. And if the active hole was activated, METH infusion was delivered through a fixed ratio 1 (FR1) reinforcement schedule. In each FR1 session, each nose-poke response in the active hole resulted in the delivery of $0.5 \mathrm{mg} / \mathrm{kg}$ per infusion METH $(0.0175 \mathrm{ml}$ injection volume delivered for 3.966 seconds) and initiated a 20 -second time-out period in which active lever presses were counted but failed to result in METH delivery. If an inactive hole was activated, there were no programmed consequences. Daily FR1 training sessions lasted 4 hours or until 200 METH infusions were self-administered in one session, whichever came first. After the acquisition, rats were allowed to self-administer METH under the FR1 and progressive ratio (PR) schedule. The PR schedule of reinforcement was introduced after the FR1 testing. Briefly, each daily PR session was 6 hours in duration. On the PR schedule, a progressively increasing number of responses $(1,2,4,6,9$, $12,15,20,25,32,40,50,62,77,95,118,145,178,219,268,328,402$, 492 , and 603) were required for the next METH infusion. Breakpoints were defined as the number of total injections after the last obtained injection, which was prior to a 1-hour period of nonreinforcement. Prior to drug testing, breakpoints maintained by METH were generated from at least three consecutive sessions of stable responding.

\section{Yoked-METH SA and Saline Procedure}

Both yoked-METH SA (yoked-METH) and yoked-Saline groups were tested simultaneously with the METH SA group in different conditions. Either a yoked-METH or a yoked-Saline rat was paired with one METH SA rat. Yoked-METH rats received the intravenous infusions of METH at the same dose, number, and rate as the METH SA group. The yoked-Saline group received the intravenous infusions of saline at the same number and rate as the METH SA group. The nose-pokes by the yoked rats were recorded but had no programmed consequences.

\section{Cue- and Drug-Induced Reinstatement of METH SA}

After METH SA, the animals were subjected to extinction for 7 days. During the extinction phase, the animals were placed in chambers, 
which were similar to those for METH SA. However, the METHassociated stimuli (cue lights or noise) were not presented, and no infusions were delivered. After extinction, the cue- or drug-induced reinstatement test was introduced. The cue-induced reinstatement test began with the noncontingent presentation of drug-associated cues, including house light and pump sound. During the experiment of cueinduced METH SA reinstatement, the light and sound cues were the same as those used during the period of METH SA acquisition. The only difference between SA and the cue-induced reinstatement test was that the drug solution was not infused during reinstatement. The drug-induced reinstatement, rats were treated with an injection of METH $(10 \mathrm{mg} / \mathrm{kg}$, i.p.). After 30 minutes, rats were placed in chambers in which METH-associated stimuli were presented and METH infusions were substituted with saline.

\section{uParaflo miRNA Microarray Assay}

A microarray assay was performed using a service provider (LC Sciences, Houston, TX) (Li et al., 2016). In the assay, RNA sample with 4-8 $\mu$ g were 3 '-extended with a poly(A) tail using poly(A) polymerase. For the following fluorescent dye staining, an oligonucleotide tag was ligated to the poly(A) tail. A microcirculation pump (Atactic Technologies, Houston, TX) was used for hybridization, which was performed overnight on a uParaflo microfluidic chip (LC Sciences). On the microfluidic chip, each detection probe consisted of a chemically modified nucleotide coding segment. The segment was complementary to the target miRNA [from miRBase (http://www.mirbase.org/)] or other RNA (control or customer-defined sequences). A spacer segment of polyethylene glycol was used to extend the coding segment away from the substrate. PGR (photogenerated reagent) chemistry was applied to prepare the detection probes via in situ synthesis. Chemical modifications of the detection probes were used to balance the hybridization melting temperatures. During the process of hybridization, 100 L6xSSPE buffer $(0.90 \mathrm{M}$ $\mathrm{NaCl}, 60 \mathrm{mM} \mathrm{Na} 2 \mathrm{HPO}_{4}$, and $6 \mathrm{mM}$ EDTA, $\mathrm{pH}$ 6.8) containing $25 \%$ formamide at $34^{\circ} \mathrm{C}$ was applied. Subsequently, tag-conjugating $\mathrm{Cy} 3$ dye was circulated through the microfluidic chip for dye staining. Fluorescence images were obtained by a laser scanner (GenePix 4000B; Molecular Devices, San Jose, CA) and analyzed by the Array-Pro Image Analysis Software (Media Cybernetics, Rockville, MD). Data were analyzed by first subtracting the background and then normalizing the signals using a LOWESS (locally weighted scatterplot smoothing) filter (for locally weighted regression).

\section{Quantitative Real-Time Polymerase Chain Reaction Analyses}

miRNA quantitative real-time polymerase chain reaction (RT-qPCR) analysis was performed using a Hairpin-it miRNA reverse-transcription PCR Quantitation Kit v1406 (GenePharma, Shanghai, People's Republic of China). All reactions were run in triplicate, and the results were normalized to those for miR-219a-5p. Amplification was performed using a reaction cycle at $95^{\circ} \mathrm{C}$ for 3 minutes, $95^{\circ} \mathrm{C}$ for 12 seconds, and $62^{\circ} \mathrm{C}$ for 40 seconds The fluorescence signal was detected at the end of each cycle. The relative miRNA production was determined using the $2^{-\Delta \mathrm{Ct}}$ method, where $\mathrm{Ct}$ is the threshold cycle.

Total RNA was extracted from Hip/VTA/NAc/prefrontal cortex (PFC) and CPU tissues, using TRIzol reagent (Invitrogen, Carlsbad, CA). cDNA was generated from $1000 \mathrm{ng}$ of mRNA with a SuperScript II Reverse Transcriptase kit and random oligonucleotides (Invitrogen). Measured mRNA abundance was normalized to $\beta$-actin and expressed as the fold change relative to the vehicle control group. All primer sequences are listed in Supplementary Table 1.

\section{Intracerebral Injection Procedures}

The animals were anesthetized using $1.5 \%$ sodium pentobarbital $(30 \mathrm{mg} / \mathrm{kg})$ and $0.03 \mathrm{ml}$ of xylazine hydrochloride. The animals were positioned in a stereotaxic frame (Ruiwoode Life Co., Ltd., Shenzhen, People's Republic of China). A total of six viral supernatant injections
( $1 \mu \mathrm{l} /$ injection; viral supernatant concentrations ranged from $6.8 \times 10^{7}$ to $1 \times 10^{8}$ infection $\mathrm{U} / \mathrm{ml}$ ) were administered on each side of the NAc. Each rat received a total of six NAc injections (Im et al., 2010). The viruses were delivered toward the core and shell portions of the NAc. The injections into the core portion of the NAc were given based on the following stereotaxic coordinates: anterior/posterior, $1.20 \mathrm{~mm}$ from the bregma; medial/lateral, \pm 1.40 and $\pm 2.40 \mathrm{~mm}$ from midline; dorsal/ventral, -6.6 and $-7.2 \mathrm{~mm}$ below the dura. The injections on the shell portion of the NAc were given based on the following stereotaxic coordinates: anterior/posterior : $1.20 \mathrm{~mm}$ from the bregma; medial/lateral, $\pm 1.40 \mathrm{~mm}$ from the midline; dorsal/ventral, $-7.9 \mathrm{~mm}$ below the dura. A small hole was drilled through the skull, and the virus was delivered through a stainless steel injection. The duration of viral supernatant injection was over 60 seconds. After the infusion, the injector remained in place for an additional 60 seconds The injector was then raised to the next more dorsal injection site, and the procedure was repeated. Finally, the holes were filled with polyethylene, and the animals were treated with penicillin sodium to prevent infection.

\section{Western Blotting}

The brains were rapidly removed after the behavioral experiments and dissected to obtain the Hip/VTA/NAc/PFC, and CPU. The tissue was homogenized on ice in an ice-cold mixture of phenylmethylsulfonyl fluoride and radioimmunoprecipitation assay lysis buffer (1:100). The homogenates were centrifuged at $16,000 \mathrm{rpm}$ at $4^{\circ} \mathrm{C}$ for 30 minutes, and the concentrations of protein were determined using the Pierce BCA Protein Assay Kit (Thermo Fisher Scientific, Waltham, MA). Equal concentrations of protein were diluted using PBS and $5 \times$ loading buffer. The protein was separated via SDS-PAGE and transferred to a PVDF membrane (BIO-RAD, Hercules, CA). The PVDF membranes were blocked in 5\% nonfat dried milk in Tris-buffered saline that contained $0.05 \%$ Tween 20 for 3 hours. The membranes were incubated at $4^{\circ} \mathrm{C}$ overnight with following primary antibodies: anti-AT1R (1:1000; ab18801; Abcam, Cambridge, UK), anti-dopamine D2 receptor (D2R, 1:5000; ab85367; Abcam), anti-CREB (1:1000; catalog number 4820; Cell Signaling Technology, Danvers, MA), anti-PLC $\beta$ ( $1: 1000 ;$ ab182359; Abcam), and anti- $\beta$-actin (1:5000; ab8226; Abcam) overnight at a temperature of $4^{\circ} \mathrm{C}$. The samples were rinsed a total of four times (each for 10 minutes) using Tris-buffered saline/Tween 20 solutions. The secondary antibody used was goat (polyclonal) antirabbit IgG (1:15,000; LI-COR, Lincoln, NE). The samples were incubated with the corresponding second antibodies at room temperature for 2 hours. The samples were also rinsed a total of four times using the Tris-buffered saline/Tween 20 solutions (10 minutes each time). The samples were developed using enhanced chemiluminescence reagent (Thermo Fisher Scientific), immunoreactivity was detected using the Odyssey Imaging System Application (LI-COR).

\section{Cell Culture}

Rat pheochromocytoma (PC12) cells were obtained from the Chinese Academy of Sciences (Shanghai, People's Republic of China) and cultured in high glucose-modified Dulbecco's modified Eagle's medium that contained $10 \% \mathrm{FBS}$ and penicillin $(100 \mathrm{U} / \mathrm{ml}) /$ streptomycin $(100 \mu \mathrm{g} / \mathrm{ml})$. The cells were cultured in an incubator at $37^{\circ} \mathrm{C}$ and $5 \%$ $\mathrm{CO}_{2}$. The culture medium was replaced every 2 days, and cells at the logarithmic growth phase were obtained for subsequent experiments.

\section{Experimental Procedures}

Experiment 1: The Effect of AT1R Blockade on METH SA and Cue-/Drug-Induced Reinstatement. After acquisition, rats self-administered METH on the FR1 and PR schedule. Five doses of METH $(0.025,0.05,0.075$, and $0.1 \mathrm{mg} / \mathrm{kg}$ per infusion) were tested using a Latin square design. Saline was given prior to DMSO and CAN testing. Rats were divided into Saline $+\operatorname{METH}(n=9), \mathrm{DMSO}+$ METH $(n=8), 5 \mathrm{CAN}(5 \mathrm{mg} / \mathrm{kg} \mathrm{CAN})+\mathrm{METH}(n=7)$, and 10CAN 
$(10 \mathrm{mg} / \mathrm{kg} \mathrm{CAN})+\operatorname{METH}(n=7)$ groups. The effects of vehicle (DMSO) administration were also examined on the day immediately prior to and after testing with one dose of CAN. Two doses of CAN (5 and $10 \mathrm{mg} / \mathrm{kg}$ ) were tested in a random order. In all tests, DMSO and CAN were administered 120 minutes prior to the initiation of SA sessions. Rats were decapitated 2 hours after saline administration, DMSO, $5 \mathrm{CAN}$, or 10CAN testing. The levels of D2R and AT1R were detected in the CPU, Hip, NAc, PFC, and VTA. After the acquisition of METH $\mathrm{SA}$, rats were maintained on $0.05 \mathrm{mg} / \mathrm{kg}$ per infusion METH under the FR1 schedule for 14 days. One additional group of rats self-administered saline during the same period of time. Based on their performance on the last 3 days of METH SA (number of infusions per session), METH SA rats were divided into five groups (METH SA, Saline, DMSO, 5CAN, and 10CAN). Rats in the METH SA were decapitated 2 hours immediately after the last SA session. The levels of D2R and AT1R were detected in the CPU, Hip, NAc, PFC, and VTA. Rats in the Saline $(n=6)$, DMSO $(n=7), 5 \mathrm{CAN}(n=7)$, and 10CAN $(n=8)$ groups underwent an additional 7 days of extinction. On the first day after extinction, each group of rats received saline, DMSO, $5 \mathrm{mg} / \mathrm{kg} \mathrm{CAN}$, or $10 \mathrm{mg} / \mathrm{kg}$ CAN, respectively. After 120 minutes, half of the rats were placed in the operant chamber for cue-induced reinstatement of METH SA. Half of rats received an intraperitoneal injection of METH 30 minutes prior to being placed in the operant chamber for druginduced reinstatement of METH SA. After an additional 7 days of abstinence, all the animals received the same vehicle or CAN on the second trial as they received in the first. Rats that were first on the cue-induced reinstatement testing were switched to drug-induced reinstatement testing, and vice versa. Rats were decapitated 2 hours after cue- or drug-induced reinstatement testing. The levels of D2R and AT1R were detected in the CPU, Hip, NAc, PFC, and VTA.

Experiment 2: The Effect of METH SA on the Expression of NAc miRNA. After METH acquisition, a total of 12 rats self-administered $0.05 \mathrm{mg} / \mathrm{kg}$ per infusion METH under the FR1 schedule for 14 days. The two additional yoked-METH $(n=12)$ and yoked-Saline $(n=12)$ groups were described previously. Two hours after the behavioral testing was completed, NAc was isolated from whole brain, and the expression of miRNA was examined. The findings of miR-219a-5p were further validated by RT-qPCR.

Experiment 3: The Effect of miRNA 219a-5p Overexpression on METH SA and Cue-/Drug-Induced Reinstatement. Rats were trained to self-administer METH, and a dose-response curve of METH SA $(0.025,0.05,0.075$, and $0.100 \mathrm{mg} / \mathrm{kg}$ per infusion) was generated as the baseline for all the animals. Based on the baseline level, the animals were further divided into two groups: rats were infused with lentiviruses as control (LV1NC, $n=6$ ) or lentiviruses containing miR-219a-5p (LV1-miR-219a-5p, $n=7$ ) into NAc and then tested on various doses of METH maintained by FR1 and PR schedules. The animals were decapitated 2 hours immediately after the SA session. Relative expression of NAc miR219a-5p was also identified in rats self-administering METH under the FR1 or PR schedule. The levels of AT1R, the primary phospholipase (PLC $\beta$ ), and CREB were also detected in the same group of animals. Additional rats self-administered $0.05 \mathrm{mg} / \mathrm{kg}$ per infusion METH under the FR1 schedule for 14 days. Based on their performance on the last 3 days of METH SA (number of infusions per session), rats were divided into two groups (LV1NC, $n=8$; LV1-miR-219a-5p, $n=7$ ). During the period of abstinence, rats were infused with LV1NC or LV1-miR-219a-5p into NAc. Half of the animals were then tested on cue-induced reinstatement of METH SA and were switched to drug-induced reinstatement of METH SA after an additional 7 days of abstinence. The other half of the animals were first tested on drug-induced reinstatement and then switched to cue-induced reinstatement of METH SA. The animals were decapitated 2 hours immediately after the reinstatement session. Relative expression of NAc miR-219a-5p was also identified in rats on either cue- or drug-induced reinstatement of METH SA. The levels of AT1R, PLC $\beta$, and CREB were also detected in the same group of animals.
Experiment 4: The Potential Role of AT1R-PLC $\beta$-CREB Signaling Pathway in the Effect of AT1R on METH SA. The relative protein expression and mRNA expression of ATR1, PLC $\beta$, and CREB was identified in rat PC12 cells. PC12 cells were seeded onto six-well plates and treated with $2.0 \mathrm{mM}$ METH and different doses of CAN $(2.5,5.0,10.0$, and $20.0 \mu \mathrm{M})$. The total RNA from PC12 cell in TRIzol reagent was determined by Multiskan Go (Thermo Fisher Scientific) after METH exposure for 24 hours. Real-time qPCR was performed according to the manufacturer instructions (AQ101; TransGen Biotech Co., Ltd, Beijing, People's Republic of China). Sequences of primers are listed in Supplemental Table 1, The PCR reaction conditions were as follows: $94^{\circ} \mathrm{C}$ for 30 seconds, 45 cycles at $94^{\circ} \mathrm{C}$ for 5 seconds, $60^{\circ} \mathrm{C}$ for 15 seconds, and $72^{\circ} \mathrm{C}$ for 10 seconds. PC12 cells were homogenized using radioimmunoprecipitation assay buffer (1:10) in which cocktail inhibitors and mercaptoethanol were freshly added. Protein concentrations were measured using the BCA Protein Assay Kit (Thermo Fisher Scientific). Twenty-five micrograms of protein was loaded on the gel. After electrophoresis, protein was transferred to a PVDF membrane and blocked with $5 \%$ bovine serum albumin or skim milk in Tris-buffered saline containing $0.1 \%$ Tween 20 for 1.5 hours. Membranes were incubated overnight with primary antibodies AT1R, PLC $\beta$, CREB, or $\beta$-actin. Secondary antibodies were incubated for 2 hours, and the blotted membranes were prepared with enhanced chemiluminescence substrate (Thermo Fisher Scientific). Band intensities were quantified by densitometry.

\section{Statistical Analysis}

All of the experimental data were presented as the mean \pm S.D. $P<0.05$ was considered as statistically significant. SPSS software (version 20.0; IBM, Armonk, NY) was used for statistical analyses. Two-way repeatedmeasures ANOVA by Bonferroni post hoc multiple-comparisons test was used to examine the effect of 5CAN and 10CAN on SA performance maintained by different doses of METH. One-way ANOVA was used to examine the effect $5 \mathrm{CAN}$ and $10 \mathrm{CAN}$ on cue- and drug-induced reinstatement of METH SA. Western blot data were expressed as ratios of D2R/ $\beta$-actin and AT1R/ $\beta$-actin. One-way ANVOA was applied to examine the changes in $\mathrm{D} 2 \mathrm{R} / \beta$-actin and AT1R/ $\beta$-actin in different brain regions. An independent $t$ test was used to compare the changes in miR-219a-5p expression among yoked-Saline, yoked-METH, and METH SA groups. Two-way repeated-measures ANOVA with Bonferroni post hoc multiple-comparisons test was used to study the effects of miR-219a-5p overexpression on SA performance maintained by different doses of METH. An independent $t$ test was used to analyze the changes in miR-219-5p expression between the LV1NC and LV1-miR-219a-5p groups. An independent $t$ test was also used to study the effects of miR-219-5p overexpression on cue- or druginduced reinstatement of METH SA and the expression of miR-219$5 p$ of LV1NC versus LV1-miR-219-59. Western blot and qPCR data were expressed as a ratio of AT1R/PLC $\beta / C R E B$. These data were analyzed by an independent $t$ test between groups.

\section{Results}

The Effect of AT1R Blockade on METH SA and Cue-/Drug-Induced Reinstatement. The effects of CAN on METH SA and cue-/drug-induced reinstatement are shown in Fig. 1. There was a major effect of Group $\left(F_{3,89}=68.819, P<\right.$ 0.001 ) (Fig. 1A), Dose $\left(F_{3,89}=28.036, P<0.001\right)$ (Fig. 1A), and Group $\times$ Dose interaction $\left(F_{6,89}=48.427, P<0.001\right)$ (Fig. 1A). Under the FR1 schedule, increasing the dose of METH significantly decreased METH SA for all four groups. Compared with Saline $+\mathrm{METH}$, DMSO $+\mathrm{METH}$, and $5 \mathrm{CAN}+\mathrm{METH}$ rats, $10 \mathrm{CAN}+\mathrm{METH}$ rats earned significantly fewer infusions of 0.025 and $0.05 \mathrm{mg} / \mathrm{kg}$ per infusion METH under the FR1 schedule $(P<0.01)$ (Fig. 1A). However, there was no major effect of CAN on the two highest doses of METH $(P>0.05)$ 

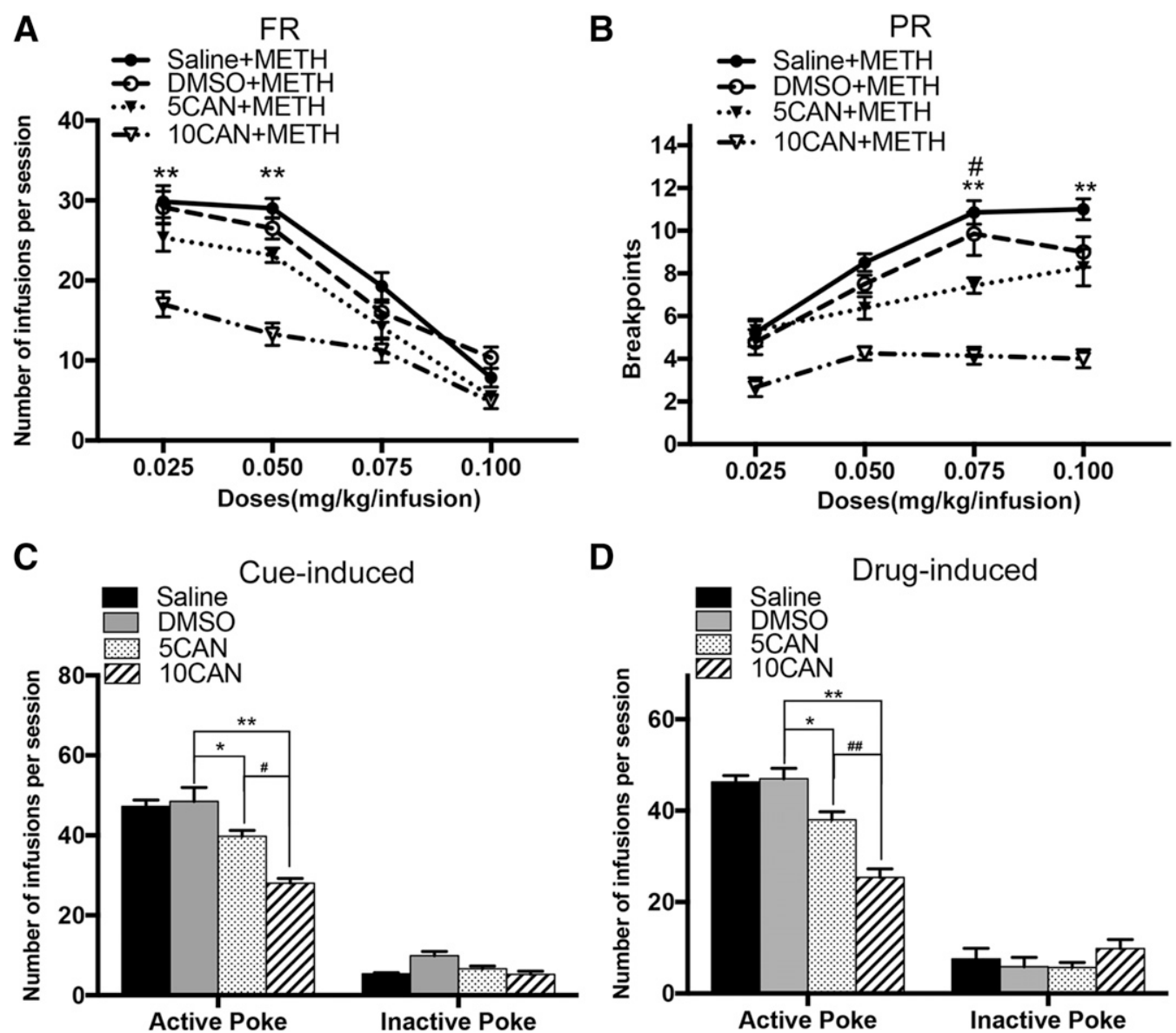

Fig. 1. The effect of AT1R blockade on METH SA and cue-/drug-induced reinstatement. (A) The effect of AT1R blockade on METH SA under the FR1 schedule. Increasing the dose of METH produced a significant decrease in drug infusions for all groups. Compared with the Saline+METH, DMSO $+\mathrm{METH}$, and 5CAN+METH groups, the 10CAN+METH group earned significantly fewer infusions of 0.025 and $0.05 \mathrm{mg} / \mathrm{kg}$ per infusion of METH after METH SA under the FR1 schedule $(* * P<0.01)$. (B) The effect of AT1R blockade on METH SA under the PR schedule. Increasing the dose of METH produced a significant increase in breakpoints for all groups. The Saline+METH and DMSO+METH groups produced significantly higher breakpoints maintained by $0.075 \mathrm{mg} / \mathrm{kg}$ per infusion METH than those of the $5 \mathrm{CAN}+\mathrm{METH}(\# P<0.05)$ and 10CAN+METH $(* * P<0.01)$ groups. Moreover, the 10CAN+METH rats produced significantly fewer breakpoints maintained by $0.1 \mathrm{mg} / \mathrm{kg}$ per infusion METH than those of the Saline+METH, DMSO + METH, and 5CAN+METH groups $(P<0.01)$. (C) The effect of AT1R blockade on cue-induced reinstatement of METH SA. Compared with the Saline and DMSO groups, the responses significantly decreased in the 5CAN $(* P<0.05)$ and 10CAN $(* * P<0.01)$ groups. The 5CAN group showed a significant decrease in response, compared with the 10 CAN group ( $\# P<0.05$ ). (D) The effect of AT1R blockade on drug-induced reinstatement of METH SA. The responses significantly decreased in the $5 \mathrm{CAN}(* P<0.05)$ and $10 \mathrm{CAN}(* * P<0.01)$ groups compared with those in the Saline and DMSO groups. The 5 CAN group showed a significant decrease in response, compared with the 10CAN group $(\# \# P<0.01)$.

(Fig. 1A). The effect of CAN on METH SA under the PR schedule is shown in Fig. 1B. There was a major effect of Group $\left(F_{3,89}=\right.$ 36.394, $P<0.01)$ (Fig. 1B), Dose $\left(F_{3,89}=19.502, P<0.01\right)$ (Fig. 1B), and Group $\times$ Dose interaction $\left(F_{6,89}=27.947, P<0.01\right)$ (Fig. 1B). Increasing the dose of METH produced a significant increase in the breakpoints for all four groups. Further post hoc analysis indicated that the Saline+METH and DMSO+ METH rats produced significantly higher breakpoints maintained by $0.075 \mathrm{mg} / \mathrm{kg}$ per infusion of METH than those of the $5 \mathrm{CAN}+\mathrm{METH}(P<0.05)($ Fig. $1 \mathrm{~B})$ and $10 \mathrm{CAN}+\mathrm{METH}$ $(P<0.01)$ (Fig. 1B) rats. In addition, 10CAN + METH rats produced significantly fewer breakpoints maintained by $0.1 \mathrm{mg} / \mathrm{kg}$ per infusion of METH than those of the Saline+ $\mathrm{METH}, \mathrm{DMSO}+\mathrm{METH}$, and $5 \mathrm{CAN}+\mathrm{METH}$ rats $(P<0.01)$ (Fig. 1B). Figure 1C illustrates the effect of CAN on the cueinduced reinstatement of METH SA. Compared with the Saline and DMSO groups, the responses significantly decreased in the 5CAN $(P<0.05)$ (Fig. 1C) and 10CAN $(P<0.01)($ Fig. 1C) groups. Relative to $5 \mathrm{CAN}, 10 \mathrm{CAN}$ produced significantly fewer responses $(P<0.05)$ (Fig. 1C). The effect of CAN on druginduced reinstatement is shown in Fig. 1D. The responses significantly decreased in the 5CAN $(P<0.05)$ (Fig. 1D) and 10CAN $(P<0.01)$ (Fig. 1D) groups, compared with those of the Saline and DMSO groups. Relative to 5CAN, 10CAN produced significantly fewer responses $(P<0.01)$ (Fig. 1D). The animals among all groups did not show significant differences in responses on inactive poke $(P>0.05)$ (Fig. 1D).

Expression of D2R and AT1R in Various Brain Regions of METH SA Rats Treated with CAN. The effect of CAN on the expression of D2R and AT1R in METH SA rats is shown in Fig. 2. One-way ANOVA showed a significant effect of Group on the level of D2R/ $\beta$-actin in VTA $\left(F_{2,8}=87.111, P<0.01\right)$ (Fig. 2A), NAc $\left(F_{2,8}=34.901, P<0.01\right)$ (Fig. 2A), and PFC $\left(F_{2,8}=75.434, P<0.01\right)$ (Fig. $\left.2 \mathrm{~A}\right)$ of rats self-administering METH maintained by the FR1 schedule. The major effect of Group was not shown in Hip and CPU. Further post hoc analysis revealed that $\mathrm{D} 2 \mathrm{R} / \beta$-actin level of group $10 \mathrm{CAN}+\mathrm{METH}$ was significantly lower than that of group DMSO + METH and 


\section{FR}

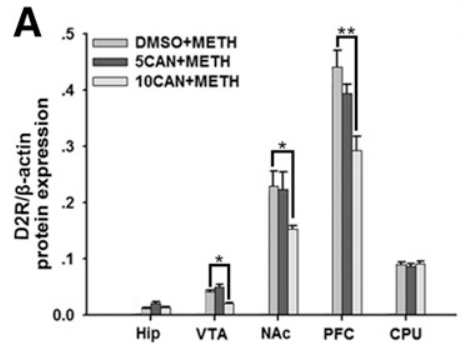

B

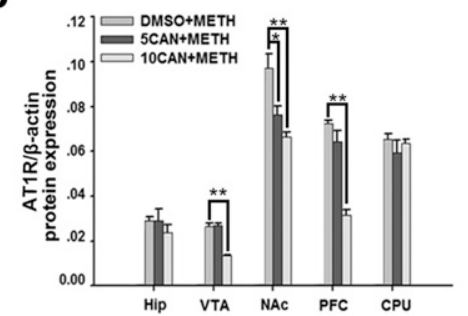

PR

D

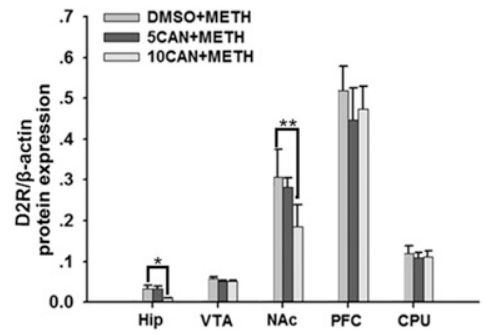

E

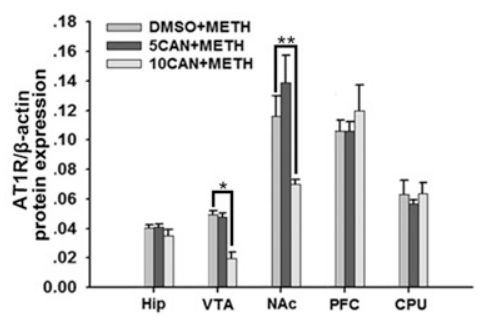

C
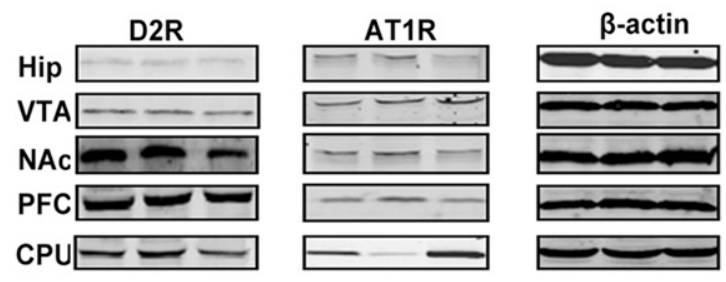

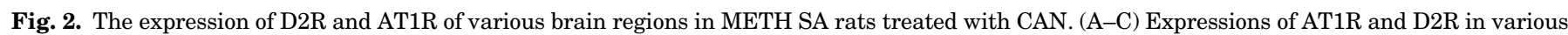

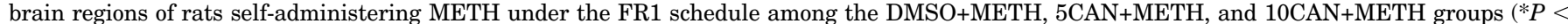

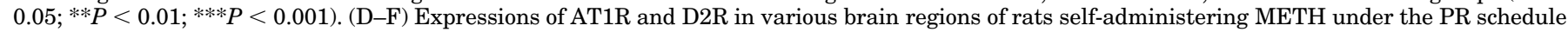
among DMSO+METH, 5CAN+METH, and 10CAN+METH groups $(* * P<0.01)$.

$5 \mathrm{CAN}+\mathrm{METH}$ in VTA, NAc, and PFC $(P<0.05)$ (Fig. 2A). There were no significant differences in $\mathrm{D} 2 \mathrm{R} / \beta$-actin expression between DMSO + METH and 5CAN + METH groups across all tested brain regions $(P>0.05)$ (Fig. $2 \mathrm{~A})$. One-way ANOVA revealed that there was a significant effect of Group on the expression of AT1R/ $\beta$-actin in VTA $\left(F_{2,8}=29.621, P<0.01\right)$ (Fig. 2B), NAc $\left(F_{2,8}=52.215, P<0.01\right)$ (Fig. $\left.2 \mathrm{~B}\right)$, and PFC $\left(F_{2,8}=43.583, P<0.01\right)$ (Fig. $\left.2 \mathrm{~B}\right)$ of rats self-administering METH maintained by the FR1 schedule. Such an effect was not evident in Hip and CPU. Further post hoc analysis revealed that VTA and PFC AT1R/ $\beta$-actin levels in the10CAN + METH group were significantly lower those of DMSO+METH and $5 \mathrm{CAN}+\mathrm{METH}$ groups $(P<0.01)$ (Fig. 2B). In NAc, the AT1R/ $\beta$-actin level of the 10CAN + METH group was significantly lower that of the DMSO+METH group $(P<0.01)$ (Fig. $2 \mathrm{~B})$. There was also a significant difference in AT1R/ $\beta$-actin levels between the METH+5CAN and DMSO+METH groups in NAc $(P<0.05)$ (Fig. 2B).

A one-way ANOVA showed a major effect of Group on D2R/ $\beta$ actin levels in Hip $\left(F_{2,8}=15.375, P<0.01\right)$ (Fig. $\left.2 \mathrm{D}\right)$ and NAc $\left(F_{2,8}=3.314, P<0.05\right)$ (Fig. $\left.2 \mathrm{D}\right)$ of rats self-administering METH maintained by the PR schedule. D2R/ $\beta$-actin level was not significantly different among groups in the brain regions of VTA, PFC, and CPU. Further post hoc testing showed that Hip and NAc D2R/ $\beta$-actin levels in $10 \mathrm{CAN}+\mathrm{METH}$ rats were significantly lower than those in the DMSO +METH and 5CAN+ METH groups $(P<0.01)$ (Fig. 2D). There was a significant effect of Group in AT1R/ $\beta$-actin expression in VTA $\left(F_{2,8}=65.979\right.$, $P<0.01)$ (Fig. 2E) and NAc $\left(F_{2,8}=21.132, P<0.01\right)$ (Fig. $\left.2 \mathrm{E}\right)$. Such an effect was not observed in Hip, PFC, and CPU. Further post hoc testing showed that in both VTA and NAc, the AT1R/ $\beta$-actin level in the $10 \mathrm{CAN}+\mathrm{METH}$ group was significantly lower than those in the DMSO+METH and 5CAN+METH groups $(P<0.01)$ (Fig. 2E).
Expression of D2R and AT1R in Various Brain Regions of Rats Treated with CAN after Cue- and Drug-Induced Reinstatement of METH SA. The effect of CAN on the expression of D2R and AT1R in METH after cue- and druginduced reinstatement of METH SA is presented in Fig. 3. There was a major effect of Group on D2R/ $\beta$-actin level in $\operatorname{VTA}\left(F_{3,15}=15.321, P<0.01\right)($ Fig. $3 \mathrm{~A}), \operatorname{NAc}\left(F_{3,15}=53.313, P<\right.$ 0.01) (Fig. 3A), and $\operatorname{PFC}\left(F_{3,15}=18.926, P<0.01\right)$ (Fig. $\left.3 \mathrm{~A}\right)$ in rats with cue-induced reinstatement of METH SA. The D2R/ $\beta$-actin level was not significantly different among groups in Hip and CPU. Further post hoc testing showed that VTA, NAc, and PFC D2R/ $\beta$-actin levels in $10 \mathrm{CAN}+\mathrm{METH}$ rats were significantly lower than those in DMSO+METH groups $(P<0.01)$ (Fig. 3A). There was a significant effect of Group in the expression of AT1R/ $\beta$-actin in Hip $\left(F_{3,15}=34.231\right.$, $P<0.01)$ (Fig. 3B), VTA $\left(F_{3,15}=6.534, P<0.01\right)$ (Fig. $\left.3 \mathrm{~B}\right)$, NAc $\left(F_{3,15}=11.42, P<0.01\right)$ (Fig. $\left.3 \mathrm{~B}\right)$, and $\mathrm{PFC}\left(F_{3,15}=\right.$ 6.321, $P<0.01$ ) (Fig. 3B). Such an effect was not observed in CPU. Further post hoc testing showed that in Hip, VTA, NAc, and PFC, AT1R/ $\beta$-actin levels in the 10CAN + METH group was significantly lower than those in the DMSO+ METH group $(P<0.01)$ (Fig. 3B).

A one-way ANOVA showed a major effect of Group on D2R/ $\beta$-actin levels in VTA $\left(F_{3,15}=4.921, P<0.01\right)$ (Fig. $\left.3 \mathrm{D}\right)$, NAc $\left(F_{3,15}=3.343, P<0.01\right)$ (Fig. $\left.3 \mathrm{D}\right)$, and $\operatorname{PFC}\left(F_{3,15}=\right.$ $14.956, P<0.01$ ) (Fig. $3 \mathrm{D})$ of rats with drug-induced reinstatement of METH SA.

$\mathrm{D} 2 \mathrm{R} / \beta$-actin level was not significantly different among groups in Hip and CPU. Further post hoc testing showed that VTA, NAc, and PFC D2R/ $\beta$-actin levels in Saline SA rats was significantly lower than that in METH SA rats $(P<0.05)$ (Fig. $3 \mathrm{D})$. VTA, NAc, and PFC D2R/ $\beta$-actin levels in the 10CAN group were also significantly lower than those in the DMSO group $(P<0.05)$ (Fig. 3D). There was a significant effect of 


\section{Cue-induced}
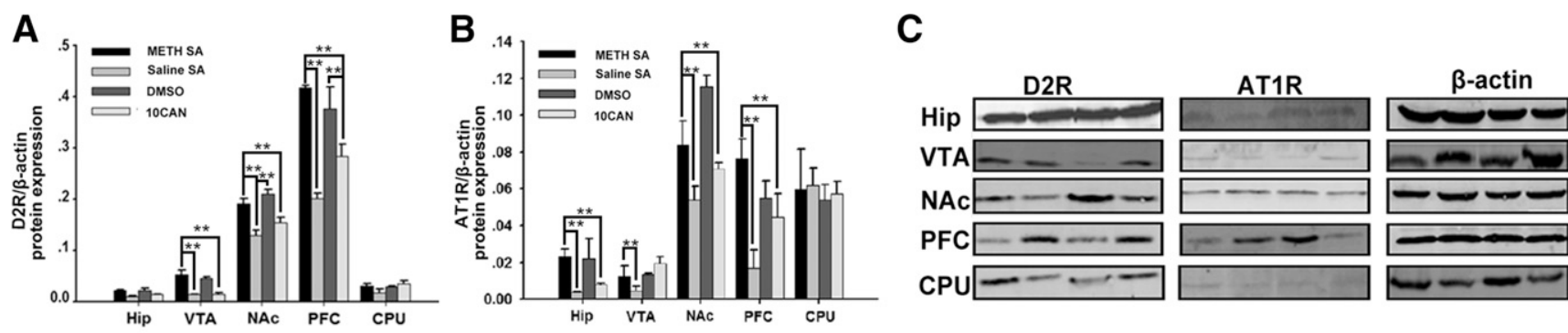

\section{Drug-induced}
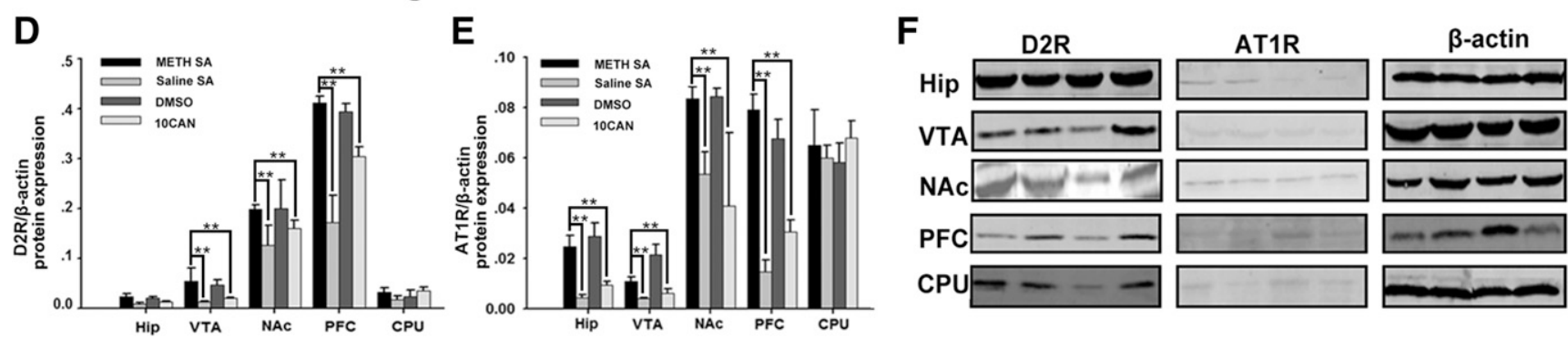

Fig. 3. The expression of $D 2 R$ and $A T 1 R$ in various brain regions of rats treated with $C A N$ under the condition of cue-induced/drug-induced reinstatement of METH SA. (A-C) Expressions of AT1R and D2R in various brain regions of rats receiving cue-induced reinstatement of the METH SA test among METH SA, Saline SA, DMSO, and 10CAN groups (** $P<0.01$ ). (D-F) Expressions of AT1R and D2R in various brain regions of rats receiving drug-induced reinstatement of METH SA test among METH SA, Saline SA, DMSO, and 10 CAN groups. AT1R and D2R proteins compared with $\beta$-actin as a standard of comparison $(* * P<0.01)$.

Group in the expression of AT1R $/ \beta$-actin in Hip $\left(F_{3,15}=34.231\right.$, $P<0.01)$ (Fig. 3E), VTA $\left(F_{3,15}=6.534, P<0.01\right)$ (Fig. $3 \mathrm{E}$ ), $\operatorname{NAc}\left(F_{3,15}=11.42, P<0.01\right)$ (Fig. $\left.3 \mathrm{E}\right)$, and $\operatorname{PFC}\left(F_{3,15}=6.321\right.$, $P<0.01$ ) (Fig. 3E). Such an effect was not observed in CPU. Further post hoc test showed that Hip, VTA, NAc, and PFC AT1R/ $\beta$-actin levels in Saline SA rats were significantly lower than those in METH SA rats $(P<0.05)$ (Fig. 3E). Hip, VTA, $\mathrm{NAc}$, and PFC AT1R/ $\beta$-actin levels in the 10CAN group were also significantly lower than those in the DMSO group $(P<0.05)$ (Fig. 3E).

The Effect of METH SA on miRNA Expression Targeting AT1R. The effects of METH SA on miRNAs are shown in Fig. 4. METH SA rats received an average of 87 infusions during the maintenance period of METH SA (Fig. 4A). Upregulated and downregulated miRNAs were identified in the yokedSaline group, yoked-METH group, and METH SA group (Fig. 4B). Colors in the heat map indicate the correlation between the different data sets. The intersection of the data were calculated by Venn diagrams and are shown by heatmap. As shown in Fig. 4C, a total of 17 miRNAs associated with RAS were significantly changed in the NAc of METH SA rats, compared with yoked-METH and yoked-Saline rats (fold change $\geq 1.5$, $P<0.05$ ). The levels of a total of eight miRNAs were upregulated; whereas, the levels of nine miRNAs were downregulated after METH SA. Compared with the yoked-METH and yokedSaline rats, miR-219a-5p was downregulated by 3.04-fold and 5.43-fold, respectively, in the NAc of METH SA rats (Fig. 4D). As shown in Fig. 4E, further independent $t$ test analysis revealed that the levels of miR-219a-5p were significantly downregulated in the NAc of METH SA rats, compared with yoked-METH and yoked-Saline rats (yokedMETH vs. METH SA, $P<0.001$; yoked-Saline vs. METH SA, $P<0.001$ ).
The Effect of miR-219a-5p Overexpression in the NAc on METH SA under the FR1 and PR Schedule. Figure 5A illustrates the effects of miR-219a-5p overexpression in the NAc on METH SA under the FR1 schedule. There was a significant effect of Group $\left(F_{2,83}=18.071, P<0.001\right)$, Dose $\left(F_{3,83}=265.736, P<0.001\right)$, and Group $\times$ Dose interaction $\left(F_{6,83}=0.618, P=0.715\right)$. Increasing the dose of METH produced a significant decrease in METH consumption for all four groups. Post hoc analysis indicated that the LV1-miR-219a-5p group earned significantly fewer infusions of $0.05,0.075$, and $0.1 \mathrm{mg} / \mathrm{kg}$ per infusion of METH than the Baseline and LV1NC groups $(0.05 \mathrm{mg} / \mathrm{kg}$ per infusion METH/LV1NC vs. LV1-miR-219a-5p, $P<0.01 ; 0.05 \mathrm{mg} / \mathrm{kg}$ per infusion METH/ Baseline vs. LV1-miR-219a-5p, $P<0.001 ; 0.075 \mathrm{mg} / \mathrm{kg}$ per infusion/METH: LV1NC vs. LV1-miR-219a-5p, $P<0.001$; $0.075 \mathrm{mg} / \mathrm{kg}$ per infusion METH/Baseline vs. LV1-miR-219a-5p, $P<0.01 ; 0.1 \mathrm{mg} / \mathrm{kg}$ per infusion/METH: LV1NC vs. LV1-miR219a-5p, $P<0.001 ; 0.1 \mathrm{mg} / \mathrm{kg}$ per infusion METH/Baseline vs. LV1-miR-219a-5p, $P<0.05)$. However, the differences for $0.025 \mathrm{mg} / \mathrm{kg}$ per infusion METH failed to reach significance among the three groups $(P>0.05)$ (Fig. 5A). As shown in Fig. 5B, the expression of NAc miR-219a-5p of the LV1-219a$5 p$ group under the FR1 schedule was significantly higher than that of the LV1NC group $(P<0.05)$.

The effects of NAc miR-219a-5p overexpression on METH SA under the PR schedule are shown in the Fig. 5C. There was a significant effect of Group $\left(F_{3,54}=49.488, P<0.001\right)$, Dose $\left(F_{2,54}=107.095, P<0.001\right)$, and a Group $\times$ Dose interaction $\left(F_{6,54}=15.390, P<0.001\right)$. Increasing the dose of METH produced a significant increase in the breakpoints for all four groups. Further post hoc analysis indicated that Baseline and LV1NC group produced significantly higher breakpoints maintained by $0.05,0.075$, and $0.1 \mathrm{mg} / \mathrm{kg}$ per infusion METH 
A

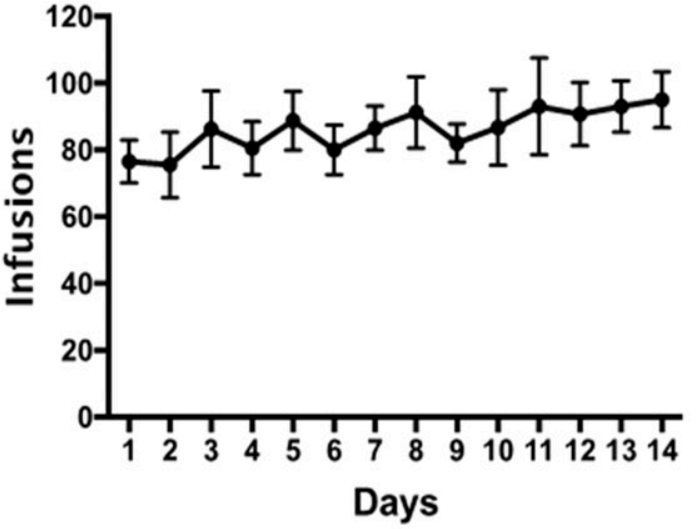

B
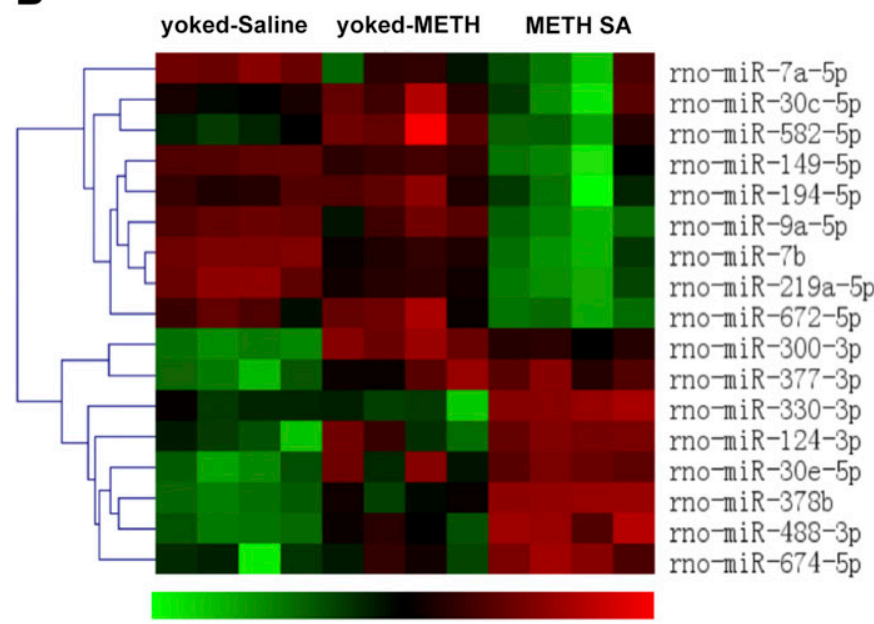

$-2.5$

0.0

2. 0

D

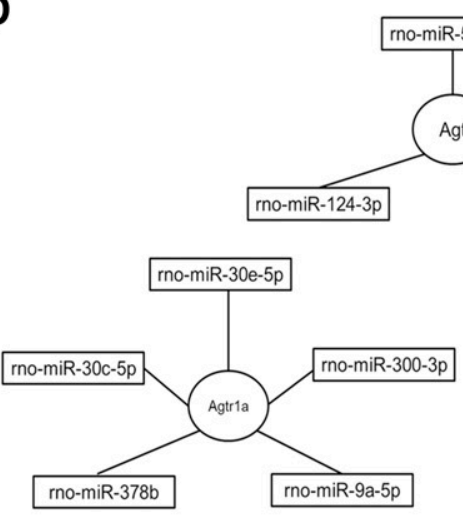

C

\begin{tabular}{|c|c|c|c|c|c|}
\hline \multirow[b]{2}{*}{$\begin{array}{l}\text { Reporter } \\
\text { Name }\end{array}$} & \multirow[b]{2}{*}{ P-Value } & \multicolumn{2}{|c|}{ METH SAIMETH-yoked } & \multicolumn{2}{|c|}{ METH SA/Saline } \\
\hline & & $\begin{array}{c}\text { Log2(METH } \\
\text { SAIMETH- } \\
\text { yoked) }\end{array}$ & $\begin{array}{c}\text { Fold } \\
\text { Change }\end{array}$ & $\begin{array}{c}\text { Log2(METH } \\
\text { SA/Saline) }\end{array}$ & $\begin{array}{c}\text { Fold } \\
\text { Change }\end{array}$ \\
\hline mo-miR-300-3p & 2.33E-08 & -0.26 & 1.19 & 0.52 & 1.43 \\
\hline mo-miR-378b & $5.79 \mathrm{E}-07$ & 0.57 & 1.49 & 0.99 & 1.98 \\
\hline mo-miR-219a-5p & $3.47 \mathrm{E}-06$ & -1.60 & 3.04 & -2.44 & 5.43 \\
\hline mo-miR-488-3p & 4.00E-05 & 0.21 & 1.15 & 0.37 & 1.29 \\
\hline mo-miR-9a-5p & 4.16E-05 & -0.62 & 1.53 & -0.71 & 1.64 \\
\hline mo-miR-672-5p & $1.54 \mathrm{E}-04$ & -0.73 & 1.65 & -0.60 & 1.51 \\
\hline mo-miR-377-3p & 2.35E-04 & 0.03 & 1.02 & 0.39 & 1.31 \\
\hline mo-miR-330-3p & 4.19E-04 & 0.69 & 1.61 & 0.51 & 1.42 \\
\hline mo-miR-30e-5p & 7.67E-04 & 0.05 & 1.03 & 0.22 & 1.16 \\
\hline mo-miR-149-5p & 1.86E-03 & -1.27 & 2.41 & -1.50 & 2.83 \\
\hline mo-miR-30c-5p & $2.49 \mathrm{E}-03$ & 0.18 & 1.13 & 0.32 & 1.25 \\
\hline mo-miR-194-5p & $3.95 \mathrm{E}-03$ & -0.42 & 1.34 & -0.36 & 1.28 \\
\hline mo-miR-582-5p & 4.00E-03 & -0.38 & 1.30 & -0.09 & 1.07 \\
\hline mo-miR-674-5p & 7.47E-03 & 0.36 & 1.28 & 0.62 & 1.53 \\
\hline mo-miR-124-3p & $1.09 \mathrm{E}-02$ & 0.13 & 1.10 & 0.25 & 1.19 \\
\hline mo-miR-7a-5p & 2.36E-02 & -0.10 & 1.07 & -0.24 & 1.18 \\
\hline mo-miR-30b-5p & 4.24E-02 & -0.14 & 1.10 & 0.07 & 1.05 \\
\hline
\end{tabular}

\section{E}

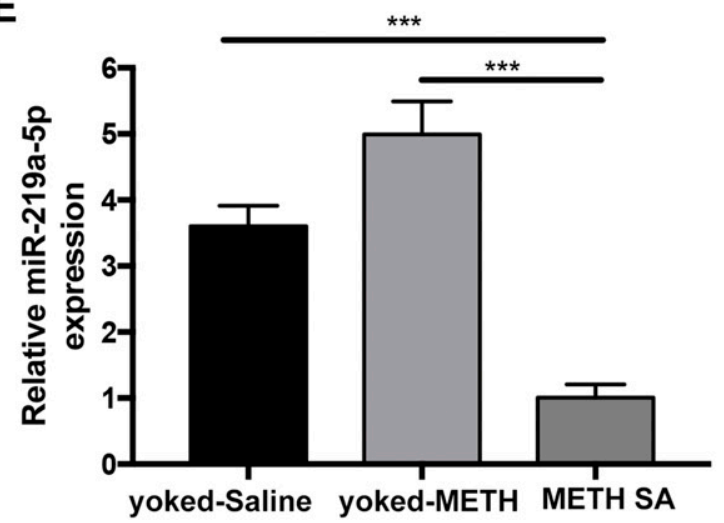

Fig. 4. The effect of METH SA on miRNA expression targeting AT1R. (A) METH SA rats exhibited stable performance of METH SA and earned an average of 87 infusions in each session. (B) A hierarchical clustering map of NAc miRNAs for METH SA, yoked-METH, and yoked-Saline rats. Colors in the heat map indicate the correlation between the different data sets. (C) The 17 miRNAs associated with RAS were significantly changed in the NAc of METH SA rats, compared with yoked-METH and yoked-Saline rats (fold change $\geq 1.5, P<0.05$ ). (D) A list of miRNAs with log2 value and fold changes. (E) miR-219a-5p expression in NAc verified by RT-qPCR (yoked-Saline group vs. yoked-METH group, ${ }^{* * *} P<0.001$; yoked-METH group vs. METH SA group, ${ }^{* * * P} P 0.001$ ).

than those of the LV1-miR219a-5p group $(0.05 \mathrm{mg} / \mathrm{kg}$ per infusion METH/LV1NC vs. LV1-miR-219a-5p, $P<0.001$ $0.05 \mathrm{mg} / \mathrm{kg}$ per infusion METH/Baseline vs. LV1-miR-219a-5p, $P<0.001 ; 0.075 \mathrm{mg} / \mathrm{kg}$ per infusion METH/LV1NC vs. LV1miR-219a-5p, $P<0.001 ; 0.075 \mathrm{mg} / \mathrm{kg}$ per infusion METH/ Baseline vs. LV1-miR-219a-5p, $P<0.001 ; 0.1 \mathrm{mg} / \mathrm{kg}$ per infusion METH/LV1NC vs. LV1-miR-219a-5p, $P<0.001 ; 0.1 \mathrm{mg} / \mathrm{kg}$ per infusion METH/Baseline vs. LV1-miR-219a-5p, $P<0.001$ ).
No differences were identified for $0.025 \mathrm{mg} / \mathrm{kg}$ per infusion METH $(P>0.05)$. As shown in Fig. 5D, the expression of NAc miR-219a-5p in the LV1-219a-5p group was significantly higher than that in the LV1NC group under the PR schedule $(P<0.001)$.

The Effect of miR-219a-5p Overexpression on Cue- and Drug-Induced Reinstatement of METH SA in NAc. The effect of miR-219a-5p overexpression in NAc on cue-induced 


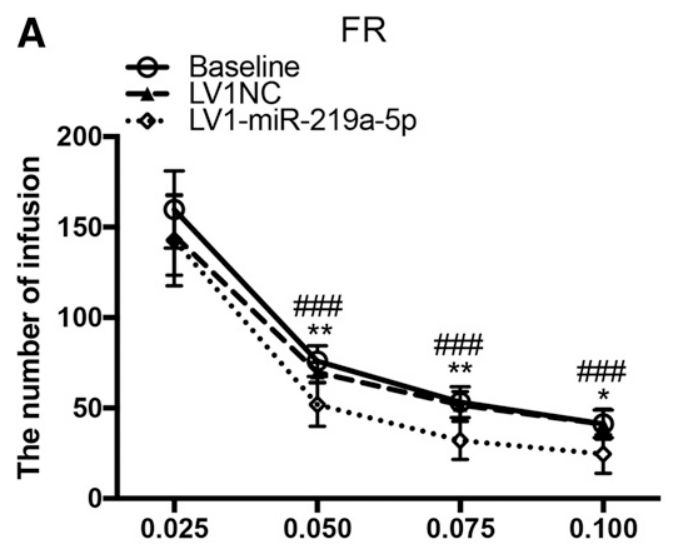

B

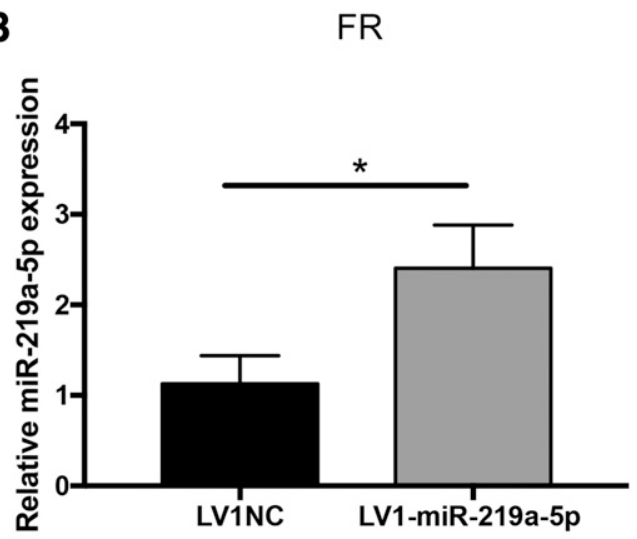

Doses (mg/kg/infusion)

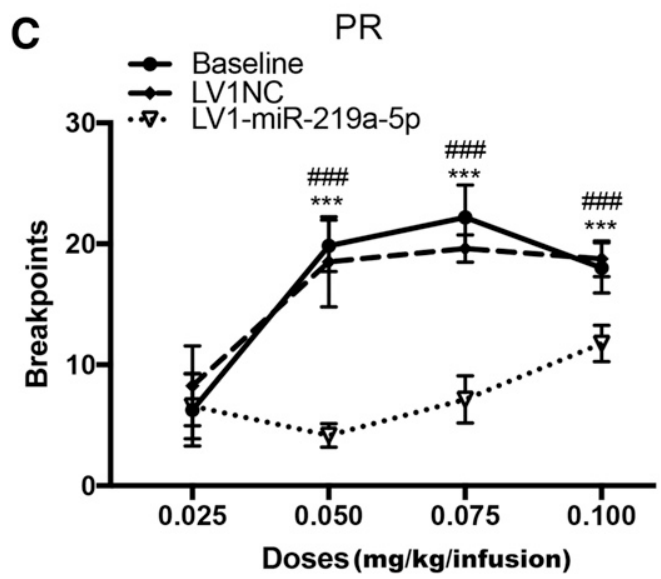

D

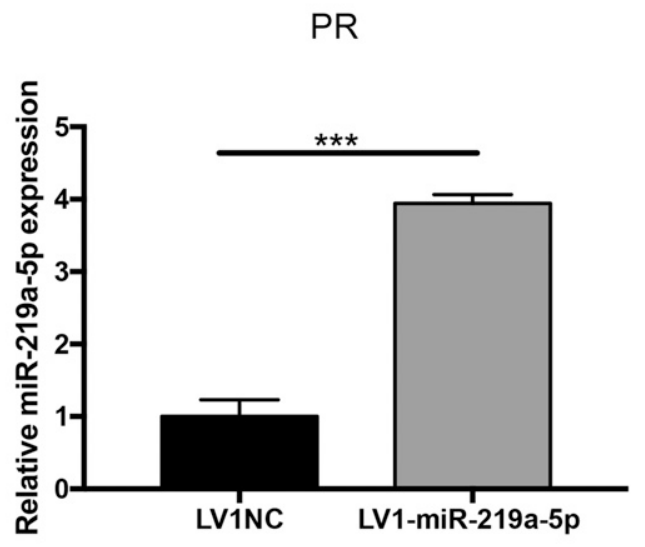

Fig. 5. The effect of miR-219a-5p overexpression in NAc on METH SA under the FR1 and PR schedule. (A) The effects of NAc miR-219a-5p overexpression on METH SA under the FR1 schedule. The LV1-miR-219a-5p group earned significantly fewer infusions of $0.05,0.075$, and $0.1 \mathrm{mg} / \mathrm{kg}$ per infusion, compared with baseline and LV1NC groups (LV1NC vs. LV1-miR-219a-5p: *** $P<0.001 ; * * P<0.01$; $* P<0.05$; baseline vs. LV1-miR-219a-5p: $\# \# \#>0.001$ ). (B) The expression of miR-219a-5p in the NAc was verified by RT-qPCR and was significantly higher in the LV1-219a-5p group than that in the LV1CN group after METH SA under the FR1 schedule (LV1NC vs. LV1-miR-219a-5p, ${ }^{*} P<0.05$ ). (C) The effects of miR-219a-5p overexpression in the NAc on METH SA under the PR schedule, compared with baseline and LV1NC groups. The LV1NC and Baseline animals produced significantly higher breakpoints maintained by $0.05,0.075$, and $0.1 \mathrm{mg} / \mathrm{kg}$ per infusion METH than those of the LV1-miR219a-5p group (LV1NC vs. LV1-miR-219a5p, $* * * P<0.001$; baseline vs. LV1-miR-219a-5p, \#\#\#P<0.001). (D) The expression of miR-219a-5p in the NAc was verified by RT-qPCR and was significantly higher in the LV1-219a-5p group than that in the LV1NC group after METH SA under the PR schedule (LV1NC vs. LV1-miR-219a-5p, $* * * P<0.001)$.

reinstatement is shown in Fig. 6A. The independent-sample $t$ test analysis showed that the LV1NC rats exhibited significantly more responses than those of the LV1-miR-219a-5p rats $(P<0.01)$. As shown in Fig. 6B for the cue-induced reinstatement of METH SA, the expression of NAc miR-219a-5p in the LV1-219a-5p rats was significantly higher than that in the LV1NC rats $(P<0.001)$. The effect of miR-219a-5p overexpression in NAc on the drug-induced reinstatement of METH SA is shown in Fig. 6C. The independent $t$ test analysis showed that the LV1-miR-219a-5p rats exhibited significantly fewer responses than those of the LV1NC rats $(P<0.01)$. As shown in Fig. 6D for the drug-induced reinstatement of METH SA, the expression of NAc miR-219a-5p in the LV1-219a-5p group was significantly higher than that in the LV1NC group $(P<0.001)$.

The Changes of AT1R, PLC $\beta$, and CREB Expression in METH SA Rats with miR-219a-5p Overexpression. Figure 7 illustrates the expression of AT1R, PLC $\beta$, and CREB proteins in LV1NC and LV1-miR-219a-5p groups after METH SA and cue-/drug-induced reinstatement. The levels of AT1R, PLC $\beta$, and CREB were significantly decreased in the LV1-miR-219a-5p rats self-administering METH under the FR1 schedule compared with the LV1NC rats $(P<0.05)$ (Fig. 7A). METH SA under the PR schedule and cue- and drug-induced reinstatement showed similar trends, as shown in Fig. 7, B-D, respectively $(P<0.05)$.

The expression of AT1R, PLC $\beta$, and CREB in PC12 cells using Western blotting was shown in Fig. 8. The expression of AT1R was increased significantly in the $2.0 \mathrm{mM}$ METH group and was markedly attenuated in the $2.5,5.0,10.0$, and $20.0 \mu \mathrm{M}$ CAN groups $(P<0.001)$ (Fig. 8B). Similarly, the level of PLC $\beta$ and CREB were also increased significantly in the $2.0 \mathrm{mM}$ METH group and decreased after treatment with 2.5, 5.0, 10.0, and $20.0 \mu \mathrm{M}$ CAN in PC12 cells $(P<0.001)$ (Fig. 8, C and D). Figure 8, E-G shows the mRNA levels of Agtr1b, PLC $\beta 1$, and CREB1 in PC12 cells. In the control PC12 cells, the mRNA levels measured by RT-qPCR were normalized by the $\beta$-actin miRNA levels and set as 1 . The treatment of $2.0 \mathrm{mM}$ METH significantly increased the levels of Agtr1b, PLC $\beta$, and CREB1 mRNA $(P<0.001)$ (Fig. 8, E-G). The increased levels of Agtr1b, PLC $\beta$, and CREB1 mRNA induced by $2.0 \mathrm{mM}$ METH were significantly decreased 
A

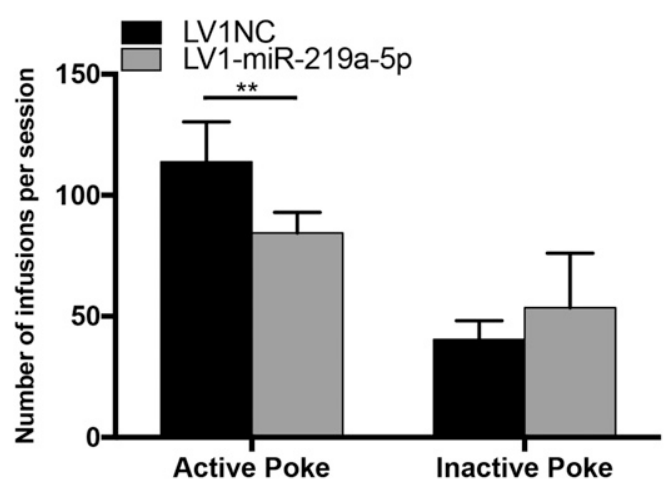

C

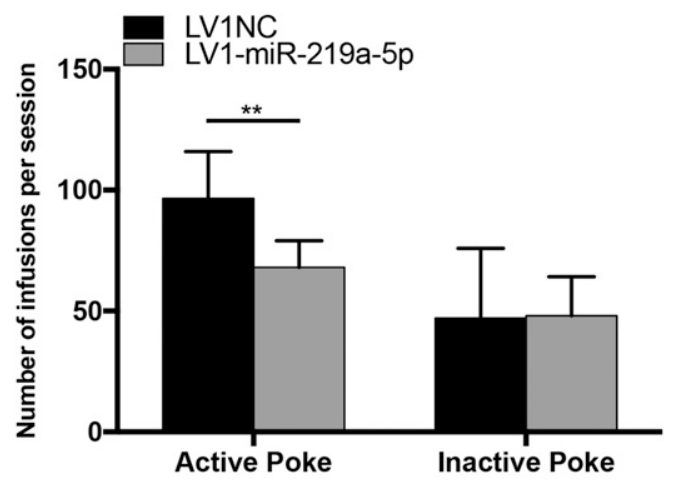

B

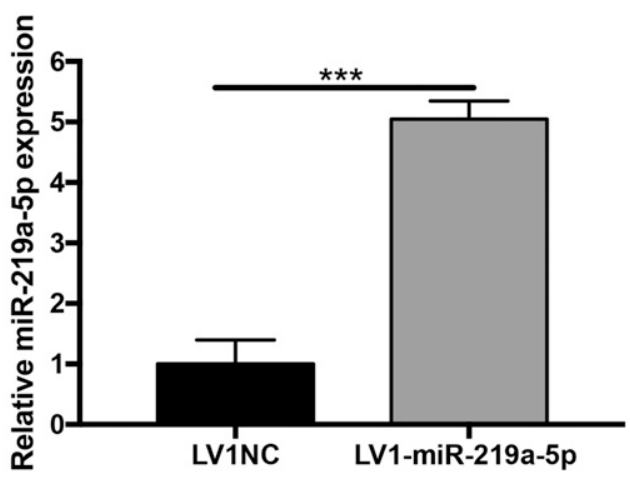

D

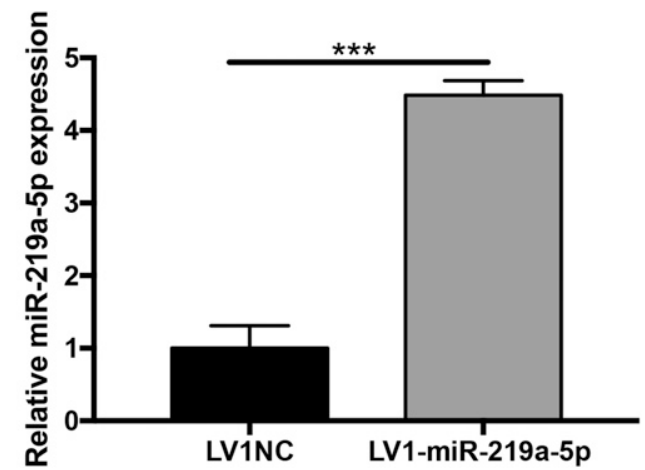

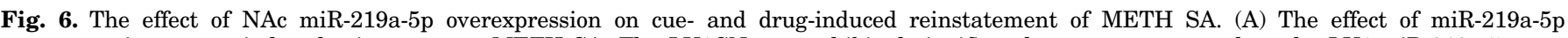

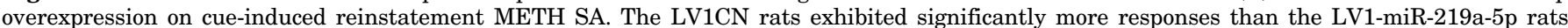

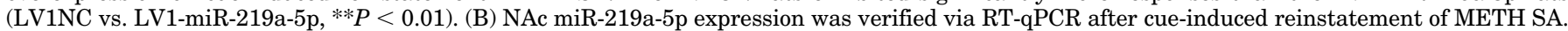

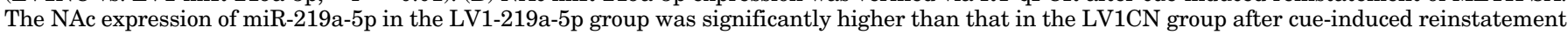

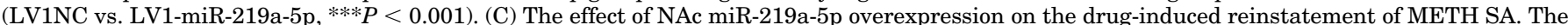

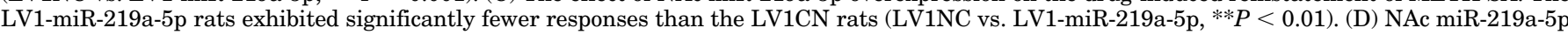

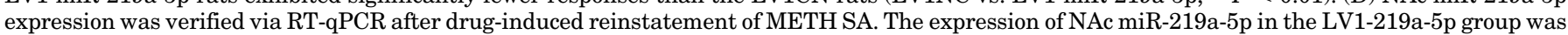
significantly higher than that in the LV1CN group after drug-induced reinstatement of METH SA (LV1NC vs. LV1-miR-219a-5p, $* * * P<0.001$ ).

by treatment with $2.5,5.0,10.0$, and $20.0 \mu \mathrm{M}$ CAN $(P<$ 0.001) (Fig. 8, E-G).

\section{Discussion}

The present study demonstrated that AT1R blockade by CAN significantly reduced METH SA and cue-/drug-induced reinstatement in a dose-dependent manner, in conjunction with altered D2R level. Involuntary intake of METH also led to a significant decrease in a total of 17 miRNAs targeting Ang and AT1R in NAc. The overexpression of NAc miR-219a-5p targeting AT1R significantly decreased METH SA under the FR1/PR schedule and cue-/drug-induced reinstatement via the AT1R-PLC $\beta$-CREB signaling pathway. Finally, our findings suggest that different subtypes of $\mathrm{PLC} \beta$ may play a distinctive role in the modulation of METH effects by AT1R blockade.

The present studies also demonstrated that the inhibition of AT1R by CAN at the dose of $10 \mathrm{mg} / \mathrm{kg}$ reduced both METH SA under the FR/PR schedule and cue-/drug- induced reinstatement. Treatment with $10 \mathrm{mg} / \mathrm{kg}$ CAN substantially reduced the elevated D2R expression induced by METH SA and reinstatement mainly in the NAc and PFC. Excess release of dopamine and dysregulation of the central dopaminergic system have largely been suggested to be responsible for the behavioral outcomes of METH (Ares-Santos et al., 2013; Courtney and Ray, 2014; Jablonski et al., 2016). Antagonistic interactions between the dopaminergic system and AT1R blockade have been described in the rat striatum and substantia nigra (Dominguez-Meijide et al., 2014). Overactivation of RAS has been shown to induce dopamine depletion, which could be prevented by AT1R blockers and angiotensin-converting enzyme inhibitors (Labandeira-Garcia et al., 2013). Overexpression of AT1R in the striatum and substantia nigra was evident in D2R-deficient mice and rats (Villar-Cheda et al., 2010). This counter-regulator effect could be explained by the functional heteromers formed by AT1R and D2R in the striatum, which was confirmed by in situ proximity ligation assay labeling (Martinez-Pinilla et al., 2015). It is unlikely that the decrease in METH SA was due to the nonspecific effects of AT1R blockade on the motor effect of METH. We examined the effects of 5 and $10 \mathrm{mg} / \mathrm{kg}$ CAN on the locomotor activity of METH. Treatment with neither 5 nor $10 \mathrm{mg} / \mathrm{kg}$ CAN significantly affected the locomotor activity of METH (unpublished data). CAN is the prodrug of candesartan. Other studies have also demonstrated that the administration of candesartan did not significantly alter the locomotor activity of rats (Gaur and Kumar, 2011). Furthermore, candersartan has been shown to improve motor dysfunction induced by stroke and thioacetamide-induced chronic 
A

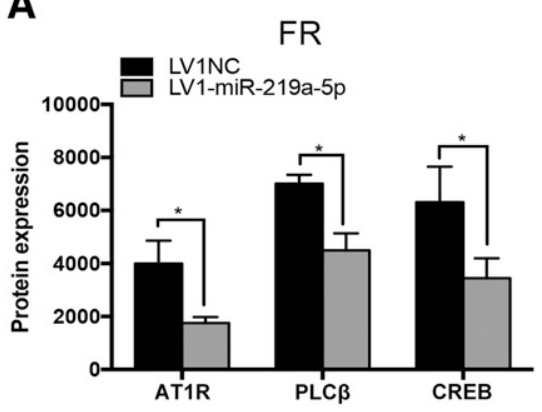

B
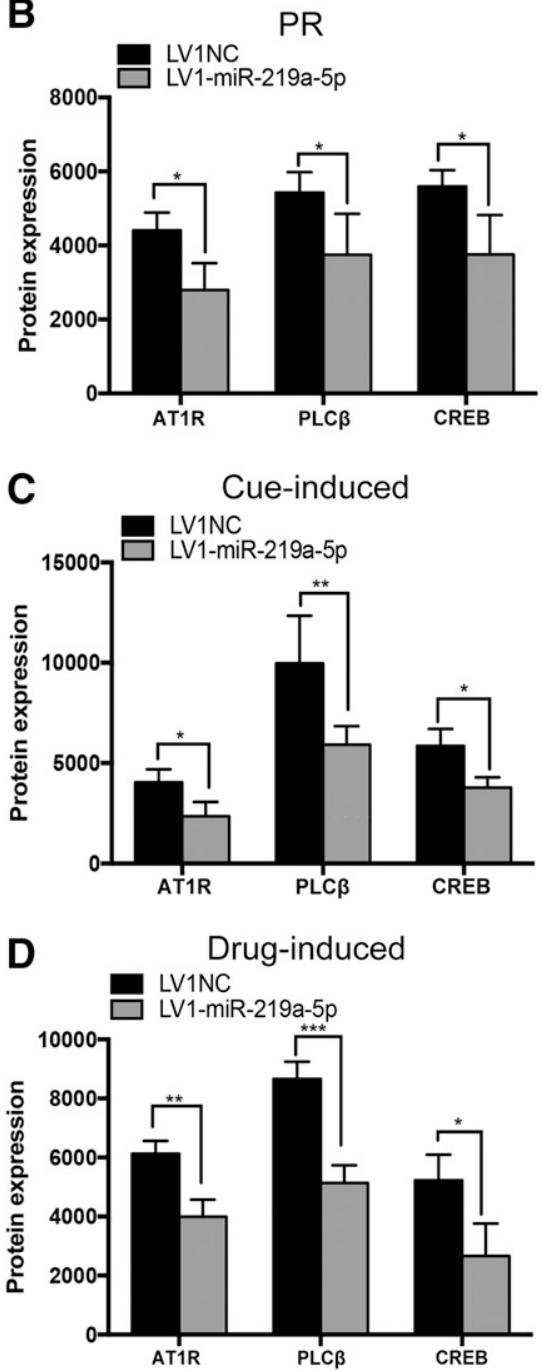
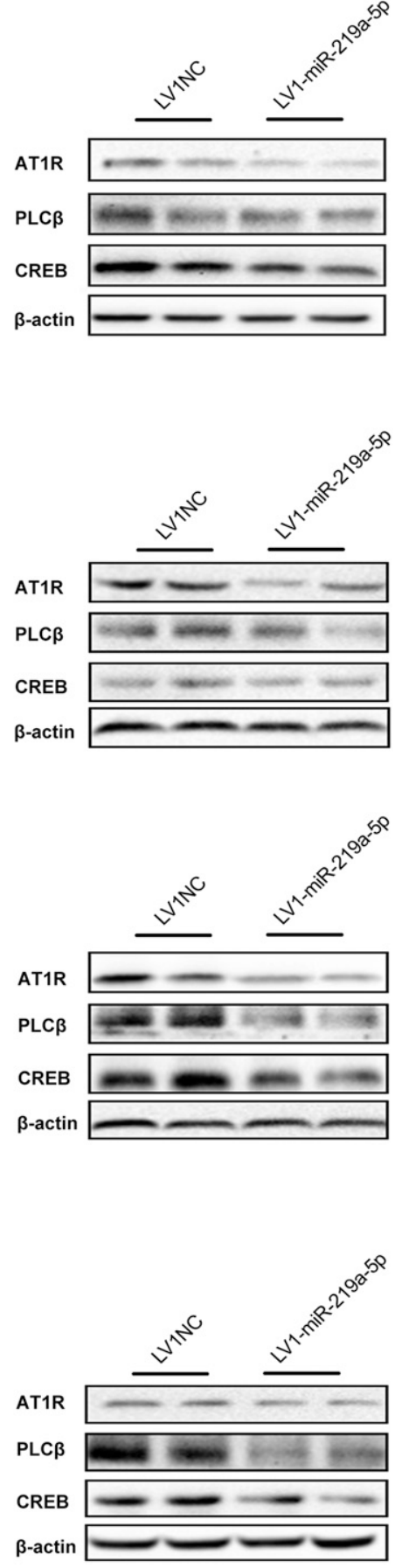

Fig. 7. The effect of the AT1R-PLC $\beta$-CREB signaling pathway on the interaction between AT1R and METH SA. (A) The levels of AT1R, PLC $\beta$, and CREB proteins were significantly decreased in rats with the overexpression of NAc miR-219a$5 p$, after METH SA under the FR1 schedule (LV1NC vs. LV1-miR-219a-5p, *P<0.05). (B) The levels of AT1R, $\operatorname{PLC} \beta$, and CREB proteins were significantly decreased in rats with the overexpression of NAc miR-219a-5p, after METH SA under the PR schedule (LV1NC vs. LV1-miR219a-5p, $* P<0.05$ ). (C) The levels of AT1R, PLC $\beta$, and CREB proteins were significantly decreased in rats with the overexpression of NAc miR-219a-5p, after cue-induced reinstatement of METH SA (LV1NC vs. LV1-miR-219a-5p $* P<0.05$; ** $P<0.01$ ). (D) The levels of AT1R, PLC- $\beta$, and CREB proteins were significantly decreased in rats with the overexpression of NAc miR-219a-5p after cue-induced reinstatement of METH SA (LV1NC vs. LV1-miR-219a-5p: $* P<0.05 ; * * P<0.01 ; * * P<0.001)$ liver failure in rats (Gaur and Kumar, 2011; Murad et al., 2017). As mentioned previously, an increasing number of studies has highlighted the potential use of medications targeting cognitive impairments associated with executive function for METH use disorder (Sofuoglu et al., 2013).

The current study demonstrated the downregulation of NAc miR-219p-5p targeting AT1R after METH SA, compared with yoked-METH and Saline rats. Current findings extend the increasing bodies of research that have demonstrated METHenabled alterations in a number of NAc miRNA expressions associated with various neuronal activities, including metabolism, apoptosis, autophagy, and immune response, potentially via the mitogen-activated protein kinase, CREB, G-protein-coupled receptor, and gonadotropin-releasing hormone signaling pathways (Zhu et al., 2015; Bai et al., 2016; Sim et al., 2017; Li et al., 2018; Zhang et al., 2018). A clinical study has also identified numerous miRNAs as negative regulators in patients with METH use disorder. The majority of studies have used the model of locomotor sensitization and conditioned place preference induced by METH that involves repeated passive administration of METH (Zhu et al., 2015; Li et al., 2018). Two recent studies have investigated the changes in miRNA in rats selfadministering METH. In both studies (Bosch et al., 2015; Du et al., 2016), control animals self-administered saline upon 
A

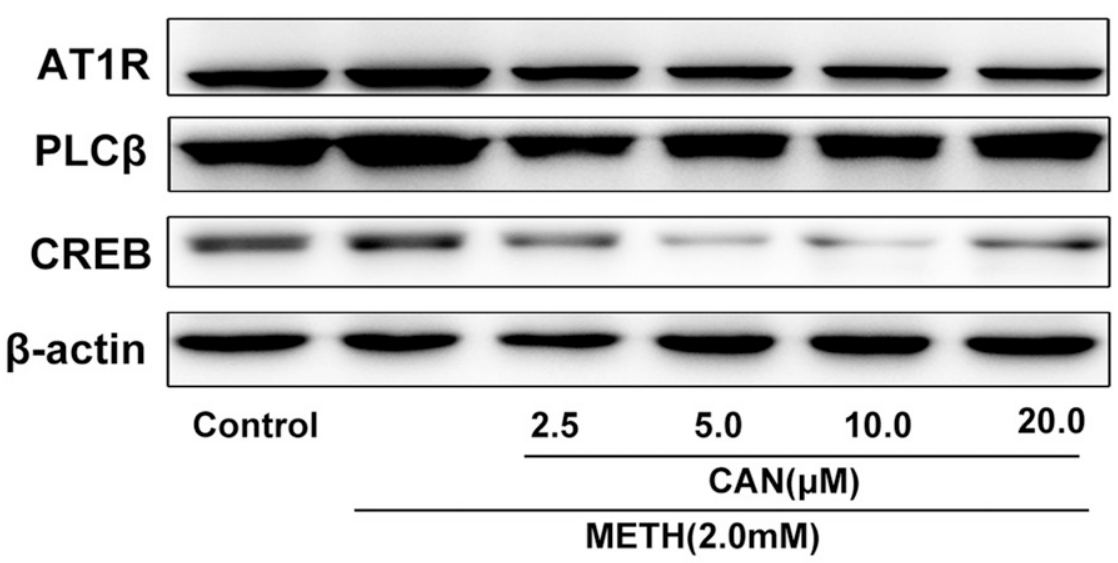

B
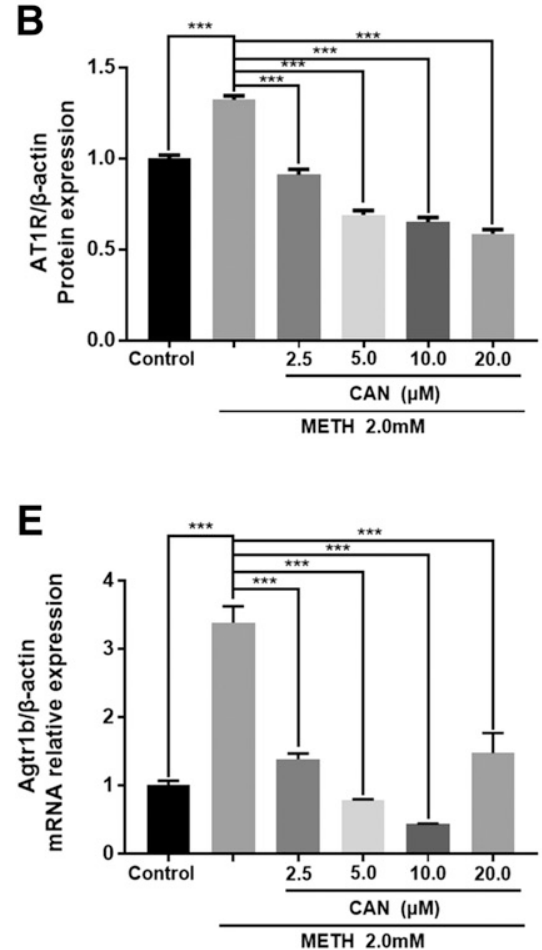

C

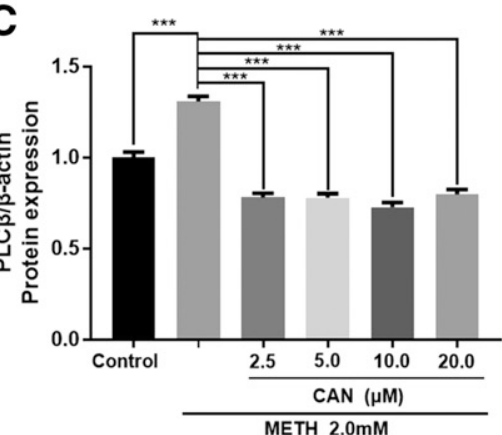

$\mathbf{F}$

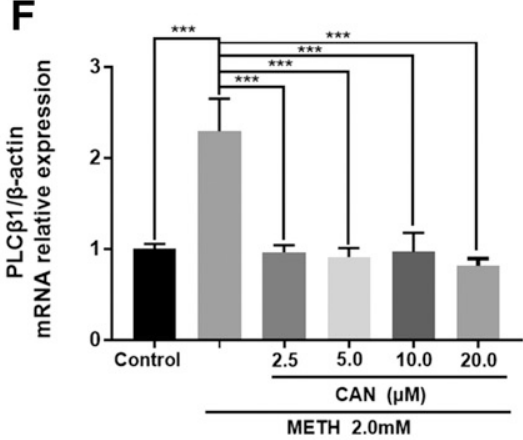

D

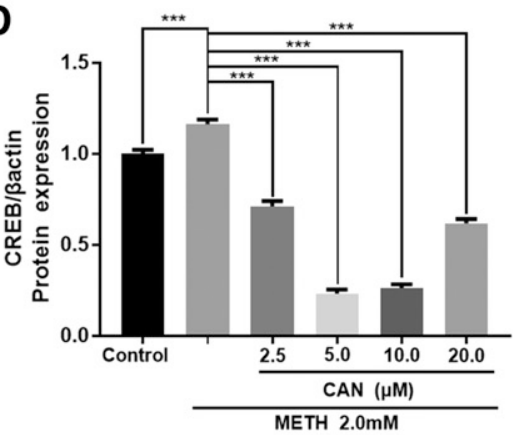

G

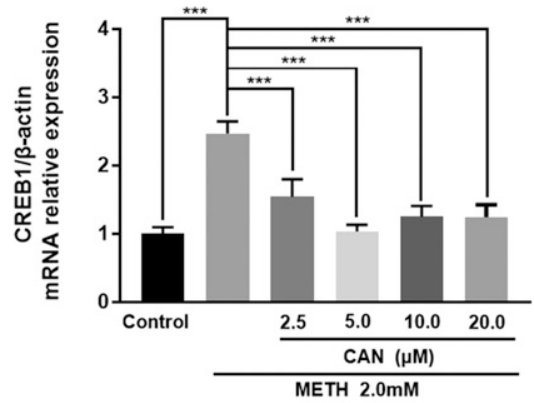

Fig. 8. Changes in AT1R/PLC $\beta /$ CREB protein and mRNA expression after METH and CAN treatment in PC12 cells. (A) Representative image of AT1R/PLC $\beta /$ CREB protein expression in PC12 cells treated with METH and CAN. (B-D) Changes in AT1R/PLC $\beta /$ CREB protein expression in PC12 cells treated with METH and CAN (***P<0.001). (E-G) mRNA levels of Agtr1b, PLC $\beta 1$, and CREB1 in PC12 cells treated with METH and CAN $(* P<$ $0.05 ; * * P<0.01 ; * * * P<0.001)$.

active responses. Twenty-eight miRNAs in the PFC and VTA were identified in rats with short and long access to METH SA, compared with animals trained in saline SA (Du et al., 2016). Seventy-eight miRNAs were differentially expressed in rats self-administering METH, compared with Saline control animals (Bosch et al., 2015). The present study identified 107 NAc miRNAs related to METH self-seeking behavior by comparing METH SA and METH-yoked rats. We further identified 17 miRNAs that targeted brain RAS, among which 3, 5, and 9 miRNAs are associated with Ang II, Agtr1a, and Agtr1b, respectively. To further investigate the altered activities of miRNAs that modulate METH SA, the effects of the overexpression of miR-219a-5p targeting AT1R were investigated with the animal model of METH SA under the FR/PR schedule and cue-/drug-induced reinstatement. MiR-219a-5p was selected because it produced the most significant $P$ values and fold changes in METH SA rats compared with the yoked-METH and yoked-Saline rats. FR and PR schedules have commonly been used to investigate the reinforcing and motivation effects of drugs of abuse (Killeen et al., 2009; Poling, 2010). The overexpression of miR-219a-5p in NAc significantly decreased METH SA at the three highest tested doses of METH under the FR and PR schedules. The potential effect of altered miR-219a-5p on cue- and drug-induced reinstatement of METH SA was subsequently examined. Similarly, the overexpression of miR-219a-5p in NAc significantly reduced the responses during both cue- and drug-induced reinstatement of $\mathrm{METH}$ SA tests.

The current study showed that the effect of AT1R on the behavioral effects of METH was associated with the changes in CREB and PLC $\beta$, along with the altered activity of D2R. There has been growing evidence about the interaction between $\mathrm{D} 2 \mathrm{R}$ and 


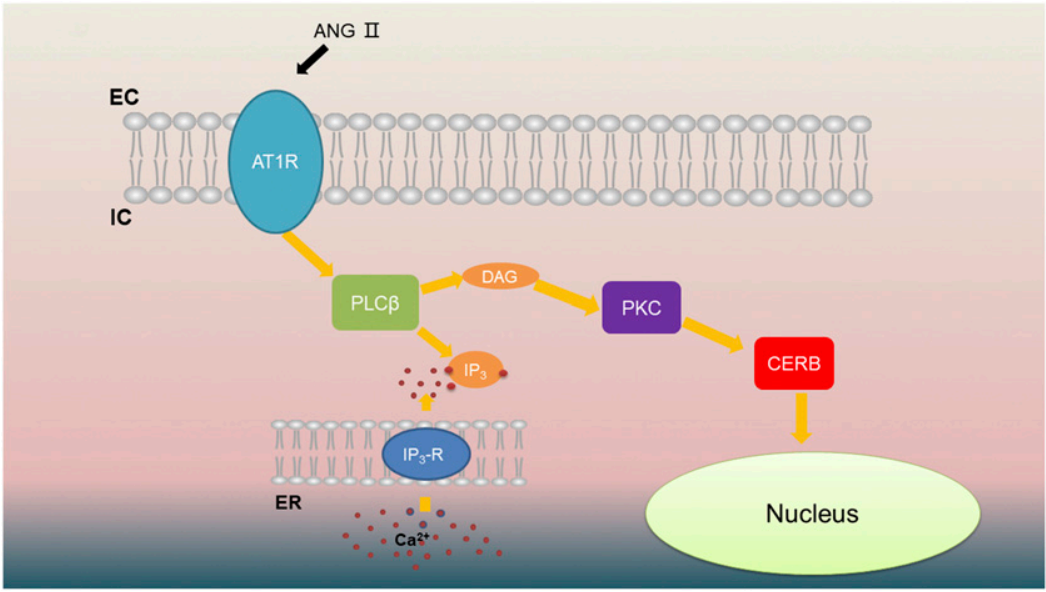

Fig. 9. The AT1R-PLC $\beta$-CREB signaling pathways. Ang II binds to AT1R. The activation of AT1R results the cleavage of IP3 and DAG from PLC $\beta$. IP 3 increases the flow of $\mathrm{Ca}^{2+}$ into the endoplasmic reticulum (ER) and increases the concentration of $\mathrm{Ca}^{2+}$. DAG activates protein kinase C (PKC), which subsequently activates CREB via phosphorylation. EC, extracellular; IC, intracellular.

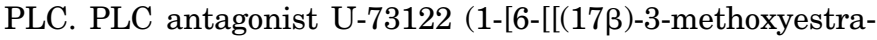
1,3,5(10)-trien-17-yl]amino]hexyl]-1H-pyrrole-2,5-dione) has been demonstrated to inhibit the effect of $\mathrm{D} 2 \mathrm{R}$ activation by quinpirole on GABAergic neurons (Jijon-Lorenzo et al., 2018). Reduced inositol monophosphate level, which indicated lower activity of PLC, was evident in cells with D2R knockdown (Simanjuntak et al., 2017). Motor skill learning deficits induced by the D2R antagonist raclopride were prevented by the coadministration of the PLC agonist m-3m3fbs (Rioult-Pedotti et al., 2015). Furthermore, the activation of PLC has also been suggested to modulate the suppressive effects of D2R stimulation on transmembrane $\mathrm{Ca}^{2+}$ currents in enkephalin-expressing medium spiny neurons (Hernandez-Lopez et al., 2000). PLC blockade by U-73122 inhibited diethylstilbestrol-induced $\mathrm{Ca}^{2+}$ mobilization and activation of CREB in gubernaculum testis cells, which suggests the underlying $\mathrm{PLC}-\mathrm{Ca}^{2+}$-CREB pathway (Zhang et al., 2018). Orexin-A dose-dependently increased $\mathrm{Ca}^{2+}$ in dopamine-containing neurons, which could be abolished by a phosphatidylcholine-specific PLC inhibitor, D609 (tricyclodecan-9-yl-xanthogenate) (Uramura et al., 2001). It is likely that PLC regulates the effects of AT1R on METH through the changes in $\mathrm{D} 2 \mathrm{R}$, and that $\mathrm{CREB} / \mathrm{Ca}^{2+}$ could be the important regulator in this process (Hamilton et al., 2015; Hille et al., 2015). PLC is classified into six isotypes, including $\beta, \gamma, \delta, \varepsilon, \zeta$, and $\eta$. $\mathrm{PLC} \beta$ is one of the most extensively studied PLCs. Due to the lack of a selective antagonist of the isotypes of PLC, it remains unknown how the specific isotypes of PLC are involved in the process. The current studies have demonstrated that the activity of PLC $\beta$ was significantly altered, in conjunction with the changes in AT1R and CREB. It is proposed that the activation of AT1R results in the cleavage of inositol triphosphate (IP3) and diacylglycerol (DAG) from PLC. IP3 increases the flow of $\mathrm{Ca}^{2+}$ from the endoplasmic reticulum and increases the concentration of $\mathrm{Ca}^{2+}$. DAG activates protein kinase $\mathrm{C}$, which subsequently activates CREB via phosphorylation (Liu et al., 2002; Song et al., 2011; Kuraishi et al., 2013; Oubrahim et al., 2013). Figure 9 illustrates the proposed link among AT1R, $\operatorname{PLC} \beta$, and CREB.

The primary PLC isozymes PLC $\beta 1$ and $\mathrm{PLC} \beta 4$ are highly expressed and differentially distributed in the brain, which suggests a specific role for each PLC subtype in different regions of the brain (Yang et al., 2016). PLC $\beta 1$ is detected at high levels in the cerebral cortex, Hip, amygdala, lateral septum, and olfactory bulb (Ross et al., 1989). Disruption of PLC $\beta 1$-mediated signaling in the brain has also been suggested to be the underlying mechanism of epilepsy, schizophrenia, and bipolar disorder (Kim et al., 1997; Garcia del Cano et al., 2014). PLC $\beta 4$ is expressed at high levels in the retina and cerebellum; however, it is rarely detected in the forebrain (Tanaka and Kondo, 1994). Dysfunction of PLC $\beta 4$ is thought to be associated with ataxia, absence seizures, and visual processing defects (Cheong et al., 2009). Furthermore, abnormal expression of PLC $\beta 4$ was identified in individuals with Huntington's disease (Cheong et al., 2009). In Experiment 4, we specifically investigated the changes in PLC $\beta 1$ and PLC $\beta 4$ in cells after the coadministration of METH and CAN. The coadministration of CAN dose-dependently prevented the increased levels of PLC $\beta 1$ and PLC $\beta 4$ by METH. It is noted that both $\mathrm{PLC} \beta 1$ and $\mathrm{PLC} \beta 4$ seem to involve the regulatory role of AT1R in METH effects. As previously discussed, METH use disorder shares common symptoms and mechanisms with a wide range of mental disorders. It is not surprising that both PLC $\beta 1$ and PLC $\beta 4$, which undermine different categories of mental disorders, are involved in METH effects.

In conclusion, the present study indicates that AT1R plays an important role in METH SA and cue-/drug-induced reinstatement of METH SA, which may provide an entirely new direction for the development of pharmacotherapeutic agents for METH use disorder.

\section{Acknowledgments}

We thank Dr. Huifen Liu for providing assistance.

\section{Authorship Contributions}

Participated in research design: Cui, $\mathrm{H}$. Xu, Zhou, and Liu.

Conducted experiments: $\mathrm{X}$. Xu, $\mathrm{Pan}, \mathrm{Li}$, and $\mathrm{Wu}$.

Performed data analysis: Mao and $\mathrm{H}$. Xu.

Wrote or contributed to the writing of the manuscript: $\mathrm{X}$. Xu, Pan, and Liu.

\section{References}

Ares-Santos S, Granado N, and Moratalla R (2013) The role of dopamine receptors in the neurotoxicity of methamphetamine. J Intern Med 273:437-453.

Bai Y, Zhang Y, Hua J, Yang X, Zhang X, Duan M, Zhu X, Huang W, Chao J, Zhou R, et al. (2016) Silencing microRNA-143 protects the integrity of the blood-brain barrier: implications for methamphetamine abuse. Sci Rep 6:35642.

Basmadjian OM, Occhieppo VB, Marchese NA, Baiardi G, and Bregonzio C (2017) Brain angiotensin II involvement in chronic mental disorders. Protein Pept Lett 24: $817-826$

Bernheim A, See RE, and Reichel CM (2016) Chronic methamphetamine selfadministration disrupts cortical control of cognition. Neurosci Biobehav Rev 69:36-48.

Bosch PJ, Benton MC, Macartney-Coxson D, and Kivell BM (2015) mRNA and microRNA analysis reveals modulation of biochemical pathways related to addiction in 
the ventral tegmental area of methamphetamine self-administering rats. $B M C$ Neurosci 16:43.

Camí J and Farré M (2003) Drug addiction. N Engl J Med 349:975-986.

Casarsa BS, Marinzalda MA, Marchese NA, Paz MC, Vivas L, Baiardi G, and Bregonzio C (2015) A previous history of repeated amphetamine exposure modifies brain angiotensin II AT1 receptor functionality. Neuroscience 307:1-13.

Cheong E, Zheng Y, Lee K, Lee J, Kim S, Sanati M, Lee S, Kim YS, and Shin HS (2009) Deletion of phospholipase $\mathrm{C}$ beta4 in thalamocortical relay nucleus leads to absence seizures. Proc Natl Acad Sci USA 106:21912-21917.

Courtney KE and Ray LA (2014) Methamphetamine: an update on epidemiology, pharmacology, clinical phenomenology, and treatment literature. Drug Alcohol Depend 143:11-21.

Dominguez-Meijide A, Villar-Cheda B, Garrido-Gil P, Sierrra-Paredes G, Guerra MJ, and Labandeira-Garcia JL (2014) Effect of chronic treatment with angiotensin type 1 receptor antagonists on striatal dopamine levels in normal rats and in a rat model of Parkinson's disease treated with L-DOPA. Neuropharmacology $\mathbf{7 6}$ (Pt A):156-168

Du HY, Cao DN, Chen Y, Wang L, Wu N, and Li J (2016) Alterations of prefrontal cortical microRNAs in methamphetamine self-administering rats: from controlled drug intake to escalated drug intake. Neurosci Lett 611:21-27.

Erlandson KM, Kitch D, Wester CW, Kalayjian RC, Overton ET, Castillo-Mancilla J, Koletar SL, Benson CA, Campbell TB, Robertson K, et al. (2017) The impact of statin and angiotensin-converting enzyme inhibitor/angiotensin receptor blocker therapy on cognitive function in adults with human immunodeficiency virus infection. Clin Infect Dis 65:2042-2049.

Fan X, Song X, Zhao M, Jarskog LF, Natarajan R, Shukair N, Freudenreich O, Henderson DC, and Goff DC (2017) The effect of adjunctive telmisartan treatment on psychopathology and cognition in patients with schizophrenia. Acta Psychiatr Scand 136:465-472.

Franke AG, Bagusat C, Rust S, Engel A, and Lieb K (2014) Substances used and prevalence rates of pharmacological cognitive enhancement among healthy subjects. Eur Arch Psychiatry Clin Neurosci 264 (Suppl 1):S83-S90.

Fulcher JA, Hussain SK, Cook R, Li F, Tobin NH, Ragsdale A, Shoptaw S, Gorbach PM, and Aldrovandi GM (2018) Effects of substance use and sex practices on the intestinal microbiome during HIV-1 infection. J Infect Dis 218:1560-1570

Gao J, Xu Z, Li X, O'Brien JW, Culshaw PN, Thomas KV, Tscharke BJ, Mueller JF, and Thai PK (2018) Enantiomeric profiling of amphetamine and methamphetamine in wastewater: a 7-year study in regional and urban Queensland, Australia. Sci Total Environ 643:827-834.

García del Caño G, Montaña M, Aretxabala X, González-Burguera I, López de Jesús M, Barrondo S, and Sallés J (2014) Nuclear phospholipase C- $\beta 1$ and diacylglycerol LIPASE- $\alpha$ in brain cortical neurons. Adv Biol Regul 54:12-23.

Gaur V and Kumar A (2011) Neuroprotective potentials of candesartan, atorvastatin and their combination against stroke induced motor dysfunction. Inflammopharmacology 19:205-214.

Grant KM, LeVan TD, Wells SM, Li M, Stoltenberg SF, Gendelman HE, Carlo G, and Bevins RA (2012) Methamphetamine-associated psychosis. J Neuroimmune Pharmacol 7:113-139.

Hamilton A, Zamponi GW, and Ferguson SS (2015) Glutamate receptors function as scaffolds for the regulation of $\beta$-amyloid and cellular prion protein signaling complexes. Mol Brain 8:18.

Härtel-Petri R, Krampe-Scheidler A, Braunwarth WD, Havemann-Reinecke U, Jeschke P, Looser W, Mühlig S, Schäfer I, Scherbaum N, Bothe L, et al. (2017) Evidence-based guidelines for the pharmacologic management of methamphetamine dependence, relapse prevention, chronic methamphetamine-related, and comorbid psychiatric disorders in post-acute settings. Pharmacopsychiatry 50:96-104.

Hernandez-Lopez S, Tkatch T, Perez-Garci E, Galarraga E, Bargas J, Hamm H, and Surmeier DJ (2000) D2 dopamine receptors in striatal medium spiny neurons reduce L-type $\mathrm{Ca} 2+$ currents and excitability via a novel PLC[beta]1-IP3-calcineurinsignaling cascade. J Neurosci 20:8987-8995.

Hille B, Dickson EJ, Kruse M, Vivas O, and Suh BC (2015) Phosphoinositides regulate ion channels. Biochim Biophys Acta 1851:844-856.

Holme MN, Rashid MH, Thomas MR, Barriga HMG, Herpoldt KL, Heenan RK, Dreiss CA, Bañuelos JL, Xie HN, Yarovsky I, et al. (2018) Fate of liposomes in the presence of phospholipase C and D: from atomic to supramolecular lipid arrangement. ACS Cent Sci 4:1023-1030.

Hosseini M, Alaei HA, Havakhah S, Neemati Karimooy HA, and Gholamnezhad Z (2009) Effects of microinjection of angiotensin II and captopril to VTA on morphine self-administration in rats. Acta Biol Hung 60:241-252.

Hosseini M, Sharifi MR, Alaei H, Shafei MN, and Karimooy HA (2007) Effects of angiotensin II and captopril on rewarding properties of morphine. Indian $J$ Exp Biol 45:770-777.

Im HI, Hollander JA, Bali P, and Kenny PJ (2010) MeCP2 controls BDNF expression and cocaine intake through homeostatic interactions with microRNA-212. Nat Neurosci 13:1120-1127.

Jablonski SA, Williams MT, and Vorhees CV (2016) Mechanisms involved in the neurotoxic and cognitive effects of developmental methamphetamine exposure. Birth Defects Res C Embryo Today 108:131-141.

Jiang L, Zhu R, Bu Q, Li Y, Shao X, Gu H, Kong J, Luo L, Long H, Guo W, et al. (2018) Brain renin-angiotensin system blockade attenuates methamphetamine-induced hyperlocomotion and neurotoxicity. Neurotherapeutics 15:500-510.

Jijón-Lorenzo R, Caballero-Florán IH, Recillas-Morales S, Cortés H, Avalos-Fuentes JA, Paz-Bermúdez FJ, Erlij D, and Florán B (2018) Presynaptic dopamine D2 receptors modulate $\left[{ }^{3} \mathrm{H}\right] \mathrm{GABA}$ release at StriatoPallidal terminals via activation of $\mathrm{PLC} \rightarrow \mathrm{IP} 3 \rightarrow$ calcineurin and inhibition of AC $\rightarrow$ CAMP $\rightarrow$ PKA signaling cascades Neuroscience 372:74-86.

Killeen PR, Posadas-Sanchez D, Johansen EB, and Thrailkill EA (2009) Progressive ratio schedules of reinforcement. J Exp Psychol Anim Behav Process 35:35-50.
Kim D, Jun KS, Lee SB, Kang NG, Min DS, Kim YH, Ryu SH, Suh PG, and Shin HS (1997) Phospholipase C isozymes selectively couple to specific neurotransmitter receptors. Nature 389:290-293.

Knuepfer MM, Rowe KD, Schwartz JA, and Lomax LL (2005) Role of angiotensin II and corticotropin-releasing hormone in hemodynamic responses to cocaine and stress. Regul Pept 127:1-10.

Krizman-Matasic I, Senta I, Kostanjevecki P, Ahel M, and Terzic S (2019) Long-term monitoring of drug consumption patterns in a large-sized European city using wastewater-based epidemiology: comparison of two sampling schemes for the assessment of multiannual trends. Sci Total Environ 647:474-485.

Kuraishi T, Hori A, and Kurata S (2013) Host-microbe interactions in the gut of Drosophila melanogaster. Front Physiol 4:375.

Labandeira-Garcia JL, Rodriguez-Pallares J, Dominguez-Meijide A, Valenzuela R, Villar-Cheda B, and Rodríguez-Perez AI (2013) Dopamine-angiotensin interactions in the basal ganglia and their relevance for Parkinson's disease. Mov Disord 28:1337-1342.

Li H, Li C, Zhou Y, Luo C, Ou J, Li J, and Mo Z (2018) Expression of microRNAs in the serum exosomes of methamphetamine-dependent rats vs. ketamine-dependent rats. Exp Ther Med 15:3369-3375.

Li H, Wang Y, Wang Z, Guo X, Wang F, Xia XJ, Zhou J, Shi K, Yu JQ, and Zhou YH (2016) Microarray and genetic analysis reveals that csa-miR159b plays a critical role in abscisic acid-mediated heat tolerance in grafted cucumber plants. Plant Cell Environ 39:1790-1804.

Liu F, Virshup DM, Nairn AC, and Greengard P (2002) Mechanism of regulation of casein kinase I activity by group I metabotropic glutamate receptors. J Biol Chem 277:45393-45399.

Martínez-Pinilla E, Rodríguez-Pérez AI, Navarro G, Aguinaga D, Moreno E, Lanciego JL, Labandeira-García JL, and Franco R (2015) Dopamine D2 and angiotensin II type 1 receptors form functional heteromers in rat striatum. Biochem Pharmacol 96:131-142.

McKetin R, Lubman DI, Baker AL, Dawe S, and Ali RL (2013) Dose-related psychotic symptoms in chronic methamphetamine users: evidence from a prospective longitudinal study. JAMA Psychiatry 70:319-324.

Mullen JM and Crawford AT (2018) Amphetamine Related Psychiatric Disorders, StatPearls, Treasure Island, FL.

Murad HA, Gazzaz ZJ, Ali SS, and Ibraheem MS (2017) Candesartan, rather than losartan, improves motor dysfunction in thioacetamide-induced chronic liver failure in rats. Braz J Med Biol Res 50:e6665.

Muthuraman A and Kaur P (2016) Renin-angiotensin-aldosterone system: a current drug target for the management of neuropathic pain. Curr Drug Targets 17:178-195.

Oubrahim H, Wong A, Wilson BA, and Chock PB (2013) Mammalian target of rapamycin complex 1 (mTORC1) plays a role in Pasteurella multocida toxin (PMT)-induced protein synthesis and proliferation in Swiss 3T3 cells. J Biol Chem 288:2805-2815

Perez-Lloret S, Otero-Losada M, Toblli JE, and Capani F (2017) Renin-angiotensin system as a potential target for new therapeutic approaches in Parkinson's disease. Expert Opin Investig Drugs 26:1163-1173.

Poling A (2010) Progressive-ratio schedules and applied behavior analysis. J Appl Behav Anal 43:347-349.

Rioult-Pedotti MS, Pekanovic A, Atiemo CO, Marshall J, and Luft AR (2015) Dopamine promotes motor cortex plasticity and motor skill learning via PLC activation. PLoS One 10:e124986.

Ross CA, MacCumber MW, Glatt CE, and Snyder SH (1989) Brain phospholipase C isozymes: differential mRNA localizations by in situ hybridization. Proc Natl Acad Sci USA 86:2923-2927.

Saavedra JM (2016) Evidence to consider angiotensin II receptor blockers for the treatment of early Alzheimer's disease. Cell Mol Neurobiol 36:259-279.

Sim MS, Soga T, Pandy V, Wu YS, Parhar IS, and Mohamed Z (2017) MicroRNA expression signature of methamphetamine use and addiction in the rat nucleus accumbens. Metab Brain Dis 32:1767-1783.

Simanjuntak Y, Liang JJ, Lee YL, and Lin YL (2017) Japanese encephalitis virus exploits dopamine D2 receptor-phospholipase $\mathrm{C}$ to target dopaminergic human neuronal cells. Front Microbiol 8:651.

Sofuoglu M, DeVito EE, Waters AJ, and Carroll KM (2013) Cognitive enhancement as a treatment for drug addictions. Neuropharmacology 64:452-463.

Song MY, Makino A, and Yuan JX (2011) Role of reactive oxygen species and redox in regulating the function of transient receptor potential channels. Antioxid Redox Signal 15:1549-1565.

Tanaka O and Kondo H (1994) Localization of mRNAs for three novel members (beta 3 , beta 4 and gamma 2 ) of phospholipase $\mathrm{C}$ family in mature rat brain. Neurosci Lett 182:17-20.

Uramura K, Funahashi H, Muroya S, Shioda S, Takigawa M, and Yada T (2001) Orexin-a activates phospholipase C- and protein kinase C-mediated $\mathrm{Ca} 2+$ signaling in dopamine neurons of the ventral tegmental area. Neuroreport 12: 1885-1889.

Vian J, Pereira C, Chavarria V, Köhler C, Stubbs B, Quevedo J, Kim SW, Carvalho AF, Berk M, and Fernandes BS (2017) The renin-angiotensin system: a possible new target for depression. BMC Med 15:144.

Villar-Cheda B, Rodríguez-Pallares J, Valenzuela R, Muñoz A, Guerra MJ, Baltatu OC, and Labandeira-Garcia JL (2010) Nigral and striatal regulation of angiotensin receptor expression by dopamine and angiotensin in rodents: implications for progression of Parkinson's disease. Eur J Neurosci 32:1695-1706.

Watanabe MA, Kucenas S, Bowman TA, Ruhlman M, and Knuepfer MM (2010) Angiotensin II and CRF receptors in the central nucleus of the amygdala mediate hemodynamic response variability to cocaine in conscious rats. Brain Res 1309:53-65.

Wright JW and Harding JW (1994) Brain angiotensin receptor subtypes in the control of physiological and behavioral responses. Neurosci Biobehav Rev 18:21-53. 
Yang YR, Kang DS, Lee C, Seok H, Follo MY, Cocco L, and Suh PG (2016) Primary phospholipase C and brain disorders. Adv Biol Regul 61:80-85.

Zhang X, Ping HY, Li JH, Duan SX, and Jiang XW (2018) Diethylstilbestrol regulates mouse gubernaculum testis cell proliferation via PLC-Ca ${ }^{2+}-\mathrm{CREB}$ pathway. Cell Biochem Funct 36:13-17.

Zhu L, Zhu J, Liu Y, Chen Y, Li Y, Chen S, Li T, Dang Y, and Chen T (2015 Chronic methamphetamine regulates the expression of MicroRNAs and putative target genes in the nucleus accumbens of mice. $J$ Neurosci Res 93:1600-1610.
Zhuang S, Wang HF, Wang X, Li J, and Xing CM (2016) The association of reninangiotensin system blockade use with the risks of cognitive impairment of aging and Alzheimer's disease: a meta-analysis. J Clin Neurosci 33:32-38.

Address correspondence to: Yu Liu, Ningbo University School of Medicine, 818 Fenghua Road, Ningbo 315211, Zhejiang, People's Republic of China. E-mail: liuyu@nbu.edu.cn 